\title{
A New Generalized Family of Odd Lindley-G Distributions With Application
}

\author{
Fastel Chipepa ${ }^{1,3}$, Broderick O. Oluyede ${ }^{1,2} \&$ Boikanyo Makubate $^{1}$ \\ 1 Department of Mathematical Statistics, Botswana International University of Science and Technology, P. Bag 16, \\ Palapye, Botswana \\ 2 Department of Mathematical Sciences, Georgia Southern University, GA 30460, USA \\ ${ }^{3}$ Department of Applied Mathematics and Statistics, Midlands State University, P. Bag 9055, Gweru, Zimbabwe \\ Correspondence: Department of Mathematical Sciences Georgia Southern University GA 30460, USA. \\ E-mail: boluyede@georgiasouthern.edu; Oluyedeo@biust.ac.bw
}

Received: August 5, 2019 Accepted: September 9, 2019 Online Published: September 25, 2019

doi:10.5539/ijsp.v8n6p1 URL: https://doi.org/10.5539/ijsp.v8n6p1

\begin{abstract}
A new family of distributions, namely the Kumaraswamy Odd Lindley-G distribution is developed. The new density function can be expressed as a linear combination of exponentiated-G densities. Statistical properties of the new family including hazard rate and quantile functions, moments and incomplete moments, Bonferroni and Lorenz curves, distribution of order statistics and Rényi entropy are derived. Some special cases are presented. We conduct some Monte Carlo simulations to examine the consistency of the maximum likelihood estimates. We provide an application of KOL-LLo distribution to a real data set.
\end{abstract}

Keywords: Kumaraswamy distribution, Lindley distribution, maximum likelihood estimation, Monte Carlo simulation, odd Lindley-G distribution

\section{Introduction}

There are considerable amount of work in the literature on the modification of the beta distribution including work by (Eugene, Lee and Famoye 2002), (Nadarajah and Kotz, 2004), (Nadarajah and Kotz, 2006), (Cordeiro, Gomes, da Silva, and Ortega, 2013), (Oluyede and Yang, 2015), and (Makubate, Oluyede, Motobetso, Huang and Fagbamigbe, 2018), to mention a few. These extended models exhibited very interesting properties because of the two extra shape parameters that make it possible to explore skewness, kurtosis and tail properties inherent in some data. The core issue with beta generated distributions is lack of tractability and this is mainly caused by the involvement of the incomplete beta function in the cumulative distribution function (cdf).

(Kumaraswamy, 1980) proposed a new distribution called the Kumaraswamy distribution. This new distribution has a wide application in hydrology. (Jones, 2009) studied the properties of the Kumaraswamy distribution and highlighted some of its similarities to the beta distribution and also its desirable tractability property over the beta distribution.

Many generators for generating extended models have been studied and these includes beta-G (B-G) by (Eugene et al., 2002), by (Cordeiro and de Castro, 2011), McDonald-G (M-G) by (Alexander, Cordeiro, Ortega and Sarabia, 2012), gamma-G type 1 by (Zografos and Balakrishnan, 2009), gamma-G type 2 (Ristić and Balakrishnan, 2012), odd-gamma-G type 3 by (Torabi and Montazari, 2012), transformed-transformer (T-X) and Weibull-X by (Alzaatreh, Lee and Famoye, 2013), exponentiated T-X by (Alzaghal, Famoye and Lee, 2013), Weibull-G by (Bourguignon, Silva and Cordeiro, 2014), Lomax-G by (Cordeiro, Ortega, Popović and Pescim, 2014) and odd Lindley-G by (Gomes-Silva, Percontini, de Brito, Ramos, Venáncio and Cordeiro, 2017), to mention a few. (Cordeiro et al, 2011) proposed a Kw-G distribution which states that given any baseline cdf $G(x)$, a new family has its cdf and pdf given by

$$
F(x)=1-\left(1-G(x)^{a}\right)^{b},
$$

and

$$
f(x)=\operatorname{abg}(x)(G(x))^{a-1}\left(1-G(x)^{a}\right)^{b-1},
$$

respectively, for $x>0, a>0$ and $b>0$.

(Gomes-Silva et al., 2017) developed the odd Lindley-G (OL-G) distribution, whose cdf and probability density function 
(pdf) are defined as

$$
F(x ; \lambda, \xi)=\int_{0}^{\frac{G(x ; \xi)}{1-G(x ; \xi)}} \frac{\lambda^{2}(1+t)}{1+\lambda} e^{-\lambda t} d t=1-\frac{\lambda+\bar{G}(x ; \xi)}{(1+\lambda) \bar{G}(x ; \xi)} \exp \left\{-\lambda \frac{G(x ; \xi)}{\bar{G}(x ; \xi)}\right\},
$$

and

$$
f(x ; \lambda, \xi)=\frac{\lambda^{2}}{(1+\lambda)} \frac{g(x ; \xi)}{[\bar{G}(x ; \xi)]^{3}} \exp \left\{-\lambda \frac{G(x ; \xi)}{\bar{G}(x ; \xi)}\right\},
$$

respectively, where $G(x ; \xi)$ and $g(x ; \xi)$ is the baseline cdf and pdf, respectively, and $\bar{G}(x ; \xi)=1-G(x ; \xi)$.

In this note, we propose a new family of distributions, namely Kumaraswamy Odd Lindley-G (KOL-G) distribution. We were motivated by some desirable properties exhibited by the Kumaraswamy distribution including simplicity of its quantile function, tractability and its easy to handle compared to the beta generated families. We hope the new distribution will attract much attention from statisticians because the distribution handles both skewness and kurtosis of data well. In section 2, we discussed the new model, the Kumaraswamy Odd Lindley-G (KOL-G) distribution. Section 3 outlines some statistical properties of the distribution including the expansion of the density function, hazard rate functions, moment, incomplete moments, moment generating and characteristic functions, mean deviation, Bonferonni and Lorenz curves, order statistics and Rényi entropy. Maximum likelihood estimation of the new model is discussed in Section 4. We discussed special cases of KOL-G distribution in section 5 and these include Kumaraswamy Odd Lindleyuniform (KOL-U), Kumaraswamy Odd Lindley-log-logistic (KOL-LLo) and Kumaraswamy Odd Lindley-Weibull (KOLW) distributions. Simulations for KOL-LLo distribution are presented in section 6. Section 7 and section 8 contain an application and conclusions, respectively.

\section{The Model}

We develop the KOL-G distribution using the generalization proposed by (Cordeiro et al., 2011), and taking $G(x)$ and $g(x)$ to be respective cdf and pdf of the Odd Lindley-G distribution by (Gomes-Silva et al., 2017). The cdf and pdf of the new KOL-G distribution are given by

$$
F_{K O L-G}(x)=1-\left(1-\left[1-\frac{\lambda+\bar{G}(x ; \xi)}{(1+\lambda) \bar{G}(x ; \xi)} \exp \left\{-\lambda \frac{G(x ; \xi)}{\bar{G}(x ; \xi)}\right\}\right]^{a}\right)^{b}
$$

and

$$
\begin{aligned}
f_{K O L-G}(x) & =a b\left[\frac{\lambda^{2}}{(1+\lambda)} \frac{g(x ; \xi)}{[\bar{G}(x ; \xi)]^{3}} \exp \left\{-\lambda \frac{G(x ; \xi)}{\bar{G}(x ; \xi)}\right\}\right] \\
& \times\left[1-\frac{\lambda+\bar{G}(x ; \xi)}{(1+\lambda) \bar{G}(x ; \xi)} \exp \left\{-\lambda \frac{G(x ; \xi)}{\bar{G}(x ; \xi)}\right\}\right]^{a-1} \\
& \times\left(1-\left[1-\frac{\lambda+\bar{G}(x ; \xi)}{(1+\lambda) \bar{G}(x ; \xi)} \exp \left\{-\lambda \frac{G(x ; \xi)}{\bar{G}(x ; \xi)}\right\}\right]^{a}\right)^{b-1},
\end{aligned}
$$

respectively, for $a, b, \lambda, \xi>0$ and $x>0$. We consider the KOL-G distribution where the baseline $\operatorname{cdf} G(x ; \xi)$ has at most two parameters. This allows us to avoid issues of overparametrization with respect to the number of parameters coming from the baseline cdf $G(x ; \xi)$.

\subsection{Sub-Classes of KOL-G Class of Distributions}

- We obtain odd Lindley-G distribution from KOL-G distribution by setting $a=b=1$.

- We also obtain a power function distribution from KOL-G distribution by setting $a=1$, with pdf given by

$$
f(x)=b\left[\frac{\lambda^{2}}{(1+\lambda)} \frac{g(x ; \xi)}{[\bar{G}(x ; \xi)]^{3}} e^{\{-\lambda \overline{G(x ; \xi)}\}}\right]\left[\frac{\lambda+\bar{G}(x ; \xi)}{(1+\lambda) \bar{G}(x ; \xi)} e^{\{-\lambda \overline{G(x ; x)}\}}\right]^{b-1} .
$$

- The exponentiated-odd Lindley-G (E-OLG) distribution is obtained by setting $b=1$, with pdf given by

$$
f(x)=a\left[\frac{\lambda^{2}}{(1+\lambda)} \frac{g(x ; \xi)}{[\bar{G}(x ; \xi)]^{3}} e^{\left\{-\lambda \frac{G(x ; \xi)}{G(x ; \xi)}\right]}\left[1-\frac{\lambda+\bar{G}(x ; \xi)}{(1+\lambda) \bar{G}(x ; \xi)} e^{\left\{-\lambda \frac{G(x ; \xi)}{G(x ; \xi)}\right]}\right]^{a-1} .\right.
$$




\section{Statistical Properties of the KOL-G Distribution}

\subsection{Expansion of the KOL-G Density}

Consider

$$
\left(1-\left[1-\frac{\lambda+\bar{G}(x ; \xi)}{(1+\lambda) \bar{G}(x ; \xi)} \exp \left\{-\lambda \frac{G(x ; \xi)}{\bar{G}(x ; \xi)}\right\}\right]^{a}\right)^{b-1}
$$

then using series expansion

$$
(1-z)^{b-1}=\sum_{m=0}^{\infty} \frac{(-1)^{m} \Gamma(b)}{\Gamma(b-m) m !} z^{m},
$$

for $|z|<1$ and $b>0$, we can write the KOL-G pdf as follows:

$$
\begin{aligned}
f_{K O L-G}(x) & =a b \sum_{m=0}^{\infty} \frac{(-1)^{m} \Gamma(b)}{\Gamma(b-m) m !}\left[\frac{\lambda^{2}}{(1+\lambda)} \frac{g(x ; \xi)}{\bar{G}(x ; \xi)^{3}} \exp \left\{-\lambda \frac{G(x ; \xi)}{\bar{G}(x ; \xi)}\right\}\right] \\
& \times\left[1-\frac{\lambda+\bar{G}(x ; \xi)}{(1+\lambda) \bar{G}(x ; \xi)} \exp \left\{-\lambda \frac{G(x ; \xi)}{\bar{G}(x ; \xi)}\right\}\right]^{a(m+1)-1} .
\end{aligned}
$$

Now, using the following series expansion

$$
\begin{aligned}
{\left[1-\frac{\lambda+\bar{G}(x ; \xi)}{(1+\lambda) \bar{G}(x ; \xi)} \exp \left\{-\lambda \frac{G(x ; \xi)}{\bar{G}(x ; \xi)}\right\}\right]^{a(m+1)-1} } & =\sum_{n=0}^{\infty} \frac{(-1)^{n} \Gamma(a(m+1))}{\Gamma(a(m+1)-n) n !} \\
& \times \frac{[\lambda+\bar{G}(x ; \xi)]^{n} \exp \left\{-\lambda n \frac{G(x ; \xi)}{\bar{G}(x ; \xi)}\right\}}{(1+\lambda)^{n} \bar{G}^{n}(x ; \xi)}
\end{aligned}
$$

we get

$$
\begin{aligned}
f_{K O L-G}(x) & =a b \sum_{m, n=0}^{\infty} \frac{(-1)^{m+n} \Gamma(b)}{\Gamma(b-m) m !} \frac{\Gamma(a(m+1))}{\Gamma(a(m+1)-n) n !} \frac{\lambda^{2}}{(1+\lambda)^{n+1}} \\
& \times \frac{[\lambda+\bar{G}(x ; \xi)]^{n}}{[\bar{G}(x ; \xi)]^{n+3}} g(x ; \xi) \exp \left\{-\lambda(n+1) \frac{G(x ; \xi)}{\bar{G}(x ; \xi)}\right\} .
\end{aligned}
$$

Applying the series expansion

$$
\exp \left\{-\lambda(n+1) \frac{G(x ; \xi)}{\bar{G}(x ; \xi)}\right\}=\sum_{q=0}^{\infty} \frac{(-1)^{q} \lambda^{q}[n+1]^{q}}{q !}\left[\frac{G(x ; \xi)}{\bar{G}(x ; \xi)}\right]^{q}
$$

and the binomial expansion

$$
[\lambda+\bar{G}(x ; \xi)]^{n}=\sum_{k=0}^{n}\left(\begin{array}{l}
n \\
k
\end{array}\right) \lambda^{n-k}[\bar{G}(x ; \xi)]^{k},
$$

we have

$$
\begin{aligned}
f_{K O L-G}(x) & =a b \sum_{q, m, n=0}^{\infty} \sum_{k=0}^{n} \frac{(-1)^{m+n+q} \Gamma(b)}{\Gamma(b-m) m !} \frac{\Gamma(a(m+1))}{\Gamma(a(m+1)-n) n !} \frac{\lambda^{2+q+n-k}[n+1]^{q}}{(1+\lambda)^{n+1}} \\
& \times\left(\begin{array}{l}
n \\
k
\end{array}\right) \frac{g(x ; \xi)}{[\bar{G}(x ; \xi)]^{n+3+q-k}}[G(x ; \xi)]^{q} .
\end{aligned}
$$

Applying the generalized binomial expansion

$$
[\bar{G}(x ; \xi)]^{-(n+3+q-k)}=\sum_{p=0}^{\infty} \frac{\Gamma(p+n+3+q-k)}{\Gamma(n+3+q-k) p !}[G(x ; \xi)]^{p},
$$

we can write KOL-G pdf as

$$
\begin{aligned}
f_{K O L-G}(x) & =a b \sum_{p, q, m, n=0}^{\infty} \sum_{k=0}^{n} \frac{(-1)^{m+n+q} \Gamma(b)}{\Gamma(b-m) m !} \frac{\Gamma(a(m+1))}{\Gamma(a(m+1)-n) n !} \frac{\lambda^{2+q+n-k}[n+1]^{q}}{(1+\lambda)^{n+1}} \\
& \times \frac{\Gamma(p+n+3+q-k)}{\Gamma(n+3+q-k) p !}\left(\begin{array}{l}
n \\
k
\end{array}\right) g(x ; \xi)[G(x ; \xi)]^{p+q} .
\end{aligned}
$$


Therefore, the KOL-G pdf can be written as

$$
\begin{aligned}
f_{K O L-G}(x) & =a b \sum_{p, q, m, n=0}^{\infty} \sum_{k=0}^{n} \frac{(-1)^{m+n+q} \Gamma(b)}{\Gamma(b-m) m !} \frac{\Gamma(a(m+1))}{\Gamma(a(m+1)-n) n !} \frac{\lambda^{2+q+n-k}[n+1]^{q}}{(1+\lambda)^{n+1}} \\
& \times \frac{\Gamma(p+n+3+q-k)}{\Gamma(n+3+q-k) p !}\left(\begin{array}{l}
n \\
k
\end{array}\right) \frac{p+q+1}{p+q+1} g(x ; \xi)[G(x ; \xi)]^{p+q} \\
& =\sum_{p, q=0}^{\infty} v_{p, q} g_{p+q}(x ; \xi),
\end{aligned}
$$

where $g_{p+q}(x ; \xi)=(p+q+1) g(x ; \xi)[G(x ; \xi)]^{p+q}$ is the exponentiated-G (Exp-G) density function with power parameter $p+q$ and

$$
\begin{aligned}
v_{p, q} & =a b \sum_{m, n=0}^{\infty} \sum_{k=0}^{n} \frac{(-1)^{m+n+q} \Gamma(b)}{\Gamma(b-m) m !} \frac{\Gamma(a(m+1))}{\Gamma(a(m+1)-n) n !} \frac{\lambda^{2+q+n-k}[n+1]^{q}}{(1+\lambda)^{n+1}} \\
& \times \frac{\Gamma(p+n+3+q-k)}{\Gamma(n+3+q-k) p !}\left(\begin{array}{l}
n \\
k
\end{array}\right) \frac{1}{p+q+1} .
\end{aligned}
$$

The KOL-G pdf is a mixture of the Exp-G densities, with parameter vector $\xi$ and power parameter $(p+q)$. Statistical properties of KOL-G distribution can be derived directly from the Exp-G family of distributions.

\subsection{Hazard Rate and Quantile Functions}

We get the hazard rate function of the KOL-G distribution by dividing KOL-G density function by its survival function.

$$
h_{K O L-G}(x)=\frac{f_{K O L-G}(x)}{\bar{F}_{K O L-G}(x)},
$$

where $f_{K O L-G}(x)$ is given by equation (4), $\bar{F}_{K O L-G}(x)=1-F_{K O L-G}(x)$ and $F_{K O L-G}(x)$ is the KOL-G cdf as in equation (3).

\subsubsection{Quantile Functions}

Note that for any fixed $\lambda>0$, and any $u \in(0,1)$, and from equation (3), the KOL-G quantile function $Q(u)$ is obtained by solving the equation

$$
1-\left(1-\left[1-\frac{\lambda+(1-G(Q(u)))}{(1+\lambda)(1-G(Q(u)))} \exp \left\{-\lambda \frac{G(Q(u))}{(1-G(Q(u)))}\right\}\right]^{a}\right)^{b}=u,
$$

so that

$$
\begin{aligned}
-\frac{\lambda+(1-G(Q(u)))}{(1-G(Q(u)))} \exp \left\{-\lambda \frac{G(Q(u))}{(1-G(Q(u)))}\right\} & =\left[\left((u-1)^{1 / b}+1\right)^{1 / a}+1\right] \\
& \times(1+\lambda) .
\end{aligned}
$$

Multiplying both sides by $e^{-(1+\lambda)}$ gives

$$
\begin{aligned}
-\frac{\lambda+(1-G(Q(u)))}{(1-G(Q(u)))} \exp \left\{-\frac{1+\lambda-G(Q(u))}{(1-G(Q(u)))}\right\} & =\left[\left((u-1)^{1 / b}+1\right)^{1 / a}+1\right] \\
& \times(1+\lambda) e^{-(1+\lambda)} .
\end{aligned}
$$

From the above equation, $-\frac{\lambda+(1-G(Q(u)))}{(1-G(Q(u))}$ is the Lambert $\mathrm{W}$ function of the real argument $\left[\left((u-1)^{1 / b}+1\right)^{1 / a}+1\right](1+$ $\lambda) e^{(-(1+\lambda))}$. The Lambert function $\mathrm{W}$, defined by $W(x) e^{W(x)}=x$ has two real branches with a branching point at $\left(-e^{-1}, 1\right)$. The lower branch, $W_{-1}(x)$, is defined in the interval $\left[-e^{-1}, 1\right]$ and has a negative singularity for $x \rightarrow 0^{-}$. The upper branch, $W_{0}(x)$, is defined for $x \in\left[-e^{-1}, \infty\right]$. It follows that,

$$
W\left(\left[\left((u-1)^{1 / b}+1\right)^{1 / a}+1\right](1+\lambda) e^{-(1+\lambda)}\right)=-\left(1+\frac{\lambda}{(1-G(Q(u)))}\right) .
$$

For any $\lambda>0$ and $u \in(0,1)$, we have $\left(1+\frac{\lambda}{(1-G(Q(u)))}\right)>1$ so that

$$
\left[\left((u-1)^{1 / b}+1^{1 / a}\right)+1\right](1+\lambda) e^{-(1+\lambda)}<0 .
$$


Now, from the lower branch of the Lambert W function, we can express equation (9) as

$$
W_{-}\left(\left[\left((u-1)^{1 / b}+1\right)^{1 / a}+1\right](1+\lambda) e^{-(1+\lambda)}\right)=-\left(1+\frac{\lambda}{(1-G(Q(u)))}\right) .
$$

Consequently, the quantile function of the KOL-G family of distributions reduces to

$$
\left.Q(u)=G^{-1}\left(1+\lambda\left[1+W_{-}\left(\left[(u-1)^{1 / b}+1\right)^{1 / a}+1\right](1+\lambda) e^{-(1+\lambda)}\right)\right]^{-1}\right) .
$$

A table of quantiles for selected parameter values of the special case of KOL-log-logistic (KOL-LLo) distribution is given in section 5 .

\subsection{Moments, Incomplete Moments, Moment Generating and Characteristic Functions}

We assume that $W_{p+q} \sim \operatorname{Exp}-\mathrm{G}(p+q)$ and let $X \sim \operatorname{KOL}-\mathrm{G}(a, b, \lambda ; \xi)$, then the $s^{\text {th }}$ moment can be obtained from equation (6) as follows:

$$
E\left(X^{s}\right)=\sum_{p, q=0}^{\infty} v_{p, q} E\left(W_{p+q}^{s}\right),
$$

where $E\left(W_{p+q}^{s}\right)$ denotes the $s^{\text {th }}$ moment of $W_{p+q}$ which follows an Exp-G distribution with parameter $(p+q)$ and $v_{p, q}$ is as defined in equation (7). Furthermore, the incomplete moments can be obtained as follows

$$
I_{X}(t)=\int_{0}^{t} x^{s} f_{K O L-G}(x ; \xi) d x=\sum_{p, q=0}^{\infty} v_{p, q} I_{p+q}(t),
$$

where $I_{p+q}(t)=\int_{0}^{t} x^{s} g_{p+q}(x ; \xi) d x$. The moment generating function (mgf) of $X$ is given by

$$
M_{X}(t)=\sum_{p, q=0}^{\infty} v_{p, q} E\left(e^{t W_{p+q}}\right)
$$

where $E\left(e^{t W_{p+q}}\right)$ is the mgf of the Exp-G distribution and $v_{p, q}$ is as defined in equation (7). Furthermore, we can obtain the characteristic function and is given by $\phi(t)=E\left(e^{i t X}\right)$, where $i=\sqrt{-1}$, that is

$$
\phi(t)=\sum_{p, q=0}^{\infty} v_{p, q} \phi_{p+q}(t),
$$

where $\phi_{p+q}(t)$ is the characteristic function of Exp-G distribution and $v_{p, q}$ is as defined in equation (7).

The coefficients of variation (CV), Skewness (CS) and Kurtosis (CK) can be readily obtained. The variance $\left(\sigma^{2}\right)$, Standard deviation $(\mathrm{SD}=\sigma)$, coefficient of variation $(\mathrm{CV})$, coefficient of skewness (CS) and coefficient of kurtosis $(\mathrm{CK})$ are given by

and

$$
\begin{gathered}
\sigma^{2}=\mu_{2}^{\prime}-\mu^{2}, \quad C V=\frac{\sigma}{\mu}=\frac{\sqrt{\mu_{2}^{\prime}-\mu^{2}}}{\mu}=\sqrt{\frac{\mu_{2}^{\prime}}{\mu^{2}}-1}, \\
C S=\frac{E\left[(X-\mu)^{3}\right]}{\left[E(X-\mu)^{2}\right]^{3 / 2}}=\frac{\mu_{3}^{\prime}-3 \mu \mu_{2}^{\prime}+2 \mu^{3}}{\left(\mu_{2}^{\prime}-\mu^{2}\right)^{3 / 2}},
\end{gathered}
$$

$$
C K=\frac{E\left[(X-\mu)^{4}\right]}{\left[E(X-\mu)^{2}\right]^{2}}=\frac{\mu_{4}^{\prime}-4 \mu \mu_{3}^{\prime}+6 \mu^{2} \mu_{2}^{\prime}-3 \mu^{4}}{\left(\mu_{2}^{\prime}-\mu^{2}\right)^{2}},
$$

respectively.

Note that the $r^{\text {th }}$ cumulant of the random variable $X$ can be readily obtained from the recursive relationship: $\kappa_{r}=\mu_{r}^{\prime}-$ $\sum_{s=1}^{r-1}\left(\begin{array}{c}r-1 \\ s-1\end{array}\right) \mu_{r-s}^{\prime} \kappa_{s}$, where $\mu_{r}^{\prime}=E\left(X-\mu_{1}^{\prime}\right)^{r}$, so that the CS and CK are given by $\gamma_{1}=\frac{\kappa_{3}}{\kappa_{2}^{3 / 2}}$ and $\gamma_{2}=\frac{\kappa_{4}}{\kappa_{2}^{2}}$. A table of moments, $\mathrm{SD}, \mathrm{CV}, \mathrm{CS}$, and CK for selected parameter values of KOL-LLo distribution are given in section 5 . 


\subsection{Mean Deviations, Bonferroni and Lorenz Curves}

Let $X \sim \operatorname{KOL-G}(a, b, \lambda, \xi)$, the mean deviation about the mean and about the median are defined by

$$
\delta_{1}(x)=\int_{0}^{\infty}|x-\mu| f_{K O L-G}(x) d x \quad \text { and } \quad \delta_{2}(x)=\int_{0}^{\infty}|x-M| f_{K O L-G}(x) d x,
$$

respectively where $\mu=E(X)$ and $M=\operatorname{Median}(X)$. The deviations can also be expressed as

$$
\begin{aligned}
\delta_{1}(x) & =2 \mu F_{K O L-G}(\mu)-2 \int_{0}^{\mu} x f_{K O L-G}(x) d x \\
& =2 \mu F_{K O L-G}(\mu)-2 \sum_{p, q=0}^{\infty} v_{p, q} I_{p+q}^{*}(t),
\end{aligned}
$$

and

$$
\delta_{2}(x)=\mu-2 \int_{0}^{M} x f_{K O L-G}(x) d x=\mu-\sum_{p, q=0}^{\infty} v_{p, q} I_{p+q}^{*}(t) .
$$

Bonferroni and Lorenz curves are given by

$$
B(p)=\frac{1}{p \mu} \int_{0}^{t} \sum_{p, q=0}^{\infty} x p_{p, q} g_{p+q}(x ; \xi) d x=\frac{1}{p \mu} \sum_{p, q=0}^{\infty} v_{p, q, m, n} I_{p+q}^{*}(t),
$$

and

$$
L(p)=\frac{1}{\mu} \int_{0}^{t} \sum_{p, q=0}^{\infty} x v_{p, q} g_{p+q}(x ; \xi) d x=\frac{1}{\mu} \sum_{p, q=0}^{\infty} v_{p, q} I_{p+q}^{*}(t),
$$

where $I_{p+q}^{*}(t)=\int_{0}^{t} x g_{p+q}(x ; \xi) d x$, is the first incomplete moment of the Exp-G distribution and $v_{p, q}$ is as given in equation (7).

\subsection{Distribution of Order Statistics}

Let $X_{1}, X_{2}, \ldots, X_{n}$ be independent and identically distributed (i.i.d) random variables distributed according to (4). The pdf of the $i^{\text {th }}$ order statistic $X_{i: n}$, is given by

$$
f_{i: n}(x)=\frac{n ! f_{K O L-G}(x)}{(i-1) !(n-i) !}\left[F_{K O L-G}(x)\right]^{i-1}\left[1-F_{K O L-G}(x)\right]^{n-i} .
$$

Substituting the KOL-G cdf, we get

$$
\begin{aligned}
f_{i: n}(x) & =\frac{n ! f_{K O L-G}(x)}{(i-1) !(n-i) !}\left[1-\left(1-\left[1-\frac{\lambda+\bar{G}(x ; \xi)}{(1+\lambda) \bar{G}(x ; \xi)} \exp \left\{-\lambda \frac{G(x ; \xi)}{\bar{G}(x ; \xi)}\right\}\right]^{a}\right)^{b}\right]^{i-1} \\
& \times\left[\left(1-\left[1-\frac{\lambda+\bar{G}(x ; \xi)}{(1+\lambda) \bar{G}(x ; \xi)} \exp \left\{-\lambda \frac{G(x ; \xi)}{\bar{G}(x ; \xi)}\right\}\right]^{a}\right)^{b}\right]^{n-i}
\end{aligned}
$$

Let

$$
z=\left(1-\left[1-\frac{\lambda+\bar{G}(x ; \xi)}{(1+\lambda) \bar{G}(x ; \xi)} \exp \left\{-\lambda \frac{G(x ; \xi)}{\bar{G}(x ; \xi)}\right\}\right]^{a}\right)^{b},
$$

then using the generalized binomial series expansion

$$
(1-z)^{i-1}=\sum_{w=0}^{\infty} \frac{(-1)^{w} \Gamma(i)}{\Gamma(i-w) w !}\left(1-\left[1-\frac{\lambda+\bar{G}(x ; \xi)}{(1+\lambda) \bar{G}(x ; \xi)} \exp \left\{-\lambda \frac{G(x ; \xi)}{\bar{G}(x ; \xi)}\right\}\right]^{a}\right)^{b w}
$$

and applying the same generalized binomial series expansion to

$$
\left(1-\left[1-\frac{\lambda+\bar{G}(x ; \xi)}{(1+\lambda) \bar{G}(x ; \xi)} \exp \left\{-\lambda \frac{G(x ; \xi)}{\bar{G}(x ; \xi)}\right\}\right]^{a}\right)^{b(n-i+w)},
$$


we have

$$
\begin{aligned}
f_{i: n}(x) & =\frac{n !}{(i-1) !(n-i) !} \sum_{j, w=0}^{\infty} \frac{(-1)^{j+w} \Gamma(i)}{\Gamma(i-w) w !} \frac{\Gamma(b(n-i+w)+1)}{\Gamma(b(n-i+w)+1-j) j !} \\
& \times\left[1-\frac{\lambda+\bar{G}(x ; \xi)}{(1+\lambda) \bar{G}(x ; \xi)} \exp \left\{-\lambda \frac{G(x ; \xi)}{\bar{G}(x ; \xi)}\right\}\right]^{a j} f_{K O L-G}(x) .
\end{aligned}
$$

Again applying the generalized binomial series expansion to

$$
\left[1-\frac{\lambda+\bar{G}(x ; \xi)}{(1+\lambda) \bar{G}(x ; \xi)} \exp \left\{-\lambda \frac{G(x ; \xi)}{\bar{G}(x ; \xi)}\right\}\right]^{a j},
$$

we can write the pdf of the $i^{\text {th }}$ order statistic as

$$
\begin{aligned}
f_{i: n}(x) & =\frac{n !}{(i-1) !(n-i) !} \sum_{k, j, w=0}^{\infty} \frac{(-1)^{k+j+w} \Gamma(i)}{\Gamma(i-w) w !} \frac{\Gamma(b(n-i+w)+1)}{\Gamma(b(n-i+w)+1-j) j !} \\
& \times \frac{\Gamma(a j+1)}{\Gamma(a j+1-k) k !} \frac{[\lambda+\bar{G}(x ; \xi)]^{k}}{(1+\lambda)^{k}[\bar{G}(x ; \xi)]^{k}} \exp \left\{-\lambda k \frac{G(x ; \xi)}{\bar{G}(x ; \xi)}\right\} f_{K O L-G}(x) .
\end{aligned}
$$

Using series expansion

$$
\exp \left\{-\lambda k \frac{G(x ; \xi)}{\bar{G}(x ; \xi)}\right\}=\sum_{z=0}^{\infty} \frac{(-1)^{z} \lambda^{z} k^{z}}{z !}\left[\frac{G(x ; \xi)}{\bar{G}(x ; \xi)}\right]^{z}
$$

we have

$$
\begin{aligned}
f_{i: n}(x) & =\frac{n !}{(i-1) !(n-i) !} \sum_{k, j, w, z=0}^{\infty} \frac{(-1)^{k+j+w+z} \lambda^{z} k^{z} \Gamma(i)}{\Gamma(i-w) w ! z !} \frac{\Gamma(b(n-i+w)+1)}{\Gamma(b(n-i+w)+1-j) j !} \\
& \times \frac{\Gamma(a j+1)}{\Gamma(a j+1-k) k !} \frac{[\lambda+\bar{G}(x ; \xi)]^{k}}{(1+\lambda)^{k} G^{\bar{k}+z}(x ; \xi)} G^{z}(x ; \xi) f_{K O L-G}(x) .
\end{aligned}
$$

Now, using binomial expansion

$$
[\lambda+\bar{G}(x ; \xi)]^{k}=\sum_{h=0}^{k}\left(\begin{array}{l}
k \\
h
\end{array}\right) \lambda^{k-h} \bar{G}^{h}(x ; \xi)
$$

and generalized binomial expansion

$$
[\bar{G}(x ; \xi)]^{-(k+z-h)}=\sum_{r=0}^{\infty} \frac{\Gamma(r+k+z-h)}{\Gamma(k+z-h) r !} G^{r}(x ; \xi),
$$

we can then write the pdf of $i^{\text {th }}$ order statistic as

$$
\begin{aligned}
f_{i: n}(x) & =\frac{n !}{(i-1) !(n-i) !} \sum_{k, j, w, r, z=0}^{\infty} \sum_{h=0}^{k} \frac{(-1)^{k+j+w+z} \lambda^{z+k-z} k^{z} \Gamma(i)}{\Gamma(i-w) w ! z !}\left(\begin{array}{l}
k \\
h
\end{array}\right) \\
& \times \frac{\Gamma(b(n-i+w)+1)}{\Gamma(b(n-i+w)+1-j) j !} \frac{\Gamma(a j+1)}{\Gamma(a j+1-k) k !} \frac{\Gamma(r+k+z-h)}{\Gamma(k+z-h) r !} \\
& \times G^{z+r}(x ; \xi) f_{K O L-G}(x) .
\end{aligned}
$$

Substituting the expansion of $f_{K O L-G}(x)$ density, we get

$$
\begin{aligned}
f_{i: n}(x) & =\frac{n !}{(i-1) !(n-i) !} \sum_{k, j, w, r, z=0}^{\infty} \sum_{p, q=0}^{\infty} \sum_{h=0}^{k} \frac{(-1)^{k+j+w+z} \lambda^{z+k-z} k^{z} \Gamma(i)}{\Gamma(i-w) w ! z !}\left(\begin{array}{l}
k \\
h
\end{array}\right) \\
& \times \frac{\Gamma(b(n-i+w)+1)}{\Gamma(b(n-i+w)+1-j) j !} \frac{\Gamma(a j+1)}{\Gamma(a j+1-k) k !} \frac{\Gamma(r+k+z-h)}{\Gamma(k+z-h) r !} \\
& \times v_{p, q} g_{p+q}(x ; \xi) G^{z+r}(x ; \xi),
\end{aligned}
$$


where $g_{p+q}(x ; \xi)=(p+q+1) g(x ; \xi)[G(x ; \xi)]^{p+q}$ such that $g_{p+q}(x ; \xi) G^{z+r}(x ; \xi)=(p+q+1) g(x ; \xi)[G(x ; \xi)]^{p+q+r+z}$, which can be written as $\frac{(p+q+1)}{(p+q+r+z+1)}(p+q+r+z+1) g(x ; \xi)[G(x ; \xi)]^{p+q+r+z}$.

Consequently, the pdf of the $i^{\text {th }}$ order statistic of KOL-G distribution can be expressed as

$$
\begin{aligned}
f_{i: n}(x) & =\frac{n !}{(i-1) !(n-i) !} \sum_{k, j, w, r, z=0}^{\infty} \sum_{p, q=0}^{\infty} \sum_{h=0}^{k} \frac{(-1)^{k+j+w+z} \lambda^{z+k-z} k^{z} \Gamma(i)}{\Gamma(i-w) w ! z !}\left(\begin{array}{l}
k \\
h
\end{array}\right) \\
& \times \frac{\Gamma(b(n-i+w)+1)}{\Gamma(b(n-i+w)+1-j) j !} \frac{\Gamma(a j+1)}{\Gamma(a j+1-k) k !} \frac{\Gamma(r+k+z-h)}{\Gamma(k+z-h) r !} \\
& \times v_{p, q} \frac{(p+q+1)}{(p+q+r+z+1)}(p+q+r+z+1) g(x ; \xi)[G(x ; \xi)]^{p+q+r+z} \\
& =\sum_{k, j, w, r, z=0}^{\infty} w_{k, j, w, r, z}^{*} g_{p+q+r+z}(x ; \xi),
\end{aligned}
$$

where $g_{p+q+r+z}(x ; \xi)=(p+q+r+z+1) g(x ; \xi)[G(x ; \xi)]^{p+q+r+z}$ is the Exp-G distribution with power parameter $(p+q+r+z)$ and

$$
\begin{aligned}
w_{k, j, w, r, z}^{*} & =\frac{n !}{(i-1) !(n-i) !} \sum_{p, q=0}^{\infty} \sum_{h=0}^{k} \frac{(-1)^{k+j+w+z} \lambda^{z+k-z} k^{z} \Gamma(i)}{\Gamma(i-w) w ! z !}\left(\begin{array}{l}
k \\
h
\end{array}\right) \\
& \times \frac{\Gamma(b(n-i+w)+1)}{\Gamma(b(n-i+w)+1-j) j !} \frac{\Gamma(a j+1)}{\Gamma(a j+1-k) k !} \frac{\Gamma(r+k+z-h)}{\Gamma(k+z-h) r !} \\
& \times v_{p, q} \frac{(p+q+1)}{(p+q+r+z+1)}
\end{aligned}
$$

Therefore, the $i^{\text {th }}$ order statistic of KOL-G distribution is a mixture of Exp-G densities with power parameter $(p+q+r+z)$. Results in equation (17) are important and can be used to find the moments of the $i^{\text {th }}$ order statistic of KOL-G distribution.

\subsection{Rényi Entropy}

Entropy measures variation of uncertainty and can be quantified by two popular measures, namely Shannon entropy by (Shannon, 1951) and Rényi entropy by (Rényi, 1961). Rényi entropy is defined by

$$
I_{R}(v)=(1-v)^{-1} \log \left[\int_{0}^{\infty} f^{v}(x ; \xi) d x\right],
$$

where $v>0$ and $v \neq 1$. We derive an expression of Rényi entropy of the KOL-G family of distributions. Substituting $f(x ; \xi)$ by KOL-G pdf and consider the following integral:

$$
\begin{aligned}
\int_{0}^{\infty} f^{v}(x ; a, b, \lambda, \xi) d x & =\frac{(a b)^{v} \lambda^{2 v}}{(1+\lambda)^{v}} \int_{0}^{\infty} \frac{g^{v}(x ; \xi)}{[\bar{G}(x ; \xi)]^{3 v}} \exp \left\{-\lambda v \frac{G(x ; \xi)}{\bar{G}(x ; \xi)}\right\} \\
& \times\left[1-\frac{\lambda+\bar{G}(x ; \xi)}{(1+\lambda) \bar{G}(x ; \xi)} \exp \left\{-\lambda \frac{G(x ; \xi)}{\bar{G}(x ; \xi)}\right\}\right]^{(a-1) v} \\
& \times\left(1-\left[1-\frac{\lambda+\bar{G}(x ; \xi)}{(1+\lambda) \bar{G}(x ; \xi)} \exp \left\{-\lambda \frac{G(x ; \xi)}{\bar{G}(x ; \xi)}\right\}\right]^{a}\right)^{(b-1) v} d x
\end{aligned}
$$

Applying the generalized binomial series expansion to

$$
\left(1-\left[1-\frac{\lambda+\bar{G}(x ; \xi)}{(1+\lambda) \bar{G}(x ; \xi)} \exp \left\{-\lambda \frac{G(x ; \xi)}{\bar{G}(x ; \xi)}\right\}\right]^{a}\right)^{(b-1) v}
$$

and

$$
\left[1-\frac{\lambda+\bar{G}(x ; \xi)}{(1+\lambda) \bar{G}(x ; \xi)} \exp \left\{-\lambda \frac{G(x ; \xi)}{\bar{G}(x ; \xi)}\right\}\right]^{(a-1) v+a m}
$$


we get

$$
\begin{aligned}
\int_{0}^{\infty} f^{v}(x ; a, b, \lambda, \xi) d x & =\frac{(a b)^{v} \lambda^{2 v}}{(1+\lambda)^{v}} \sum_{m, n=0}^{\infty} \frac{(-1)^{m+n} \Gamma((b-1) v+1)}{\Gamma((b-1) v-m) m !(1+\lambda)^{n+v}} \\
& \times \frac{\Gamma((a-1) v+a m+1)}{\Gamma((a-1) v+a m+1-n) n !} \int_{0}^{\infty} \frac{[\lambda+\bar{G}(x ; \xi)]^{n}}{[\bar{G}(x ; \xi)]^{n+3 v}} \\
& \times g^{v}(x ; \xi) \exp \left\{-\lambda(v+n) \frac{G(x ; \xi)}{\bar{G}(x ; \xi)}\right\} d x .
\end{aligned}
$$

Using the series expansion

$$
\exp \left\{-\lambda(n+v) \frac{G(x ; \xi)}{\bar{G}(x ; \xi)}\right\}=\sum_{q=0}^{\infty} \frac{(-1)^{q} \lambda^{q}[n+v]^{q}}{q !}\left[\frac{G(x ; \xi)}{\bar{G}(x ; \xi)}\right]^{q},
$$

binomial expansion followed by generalized binomial expansion

$$
[\lambda+\bar{G}(x ; \xi)]^{n}=\sum_{k=0}^{n}\left(\begin{array}{l}
n \\
k
\end{array}\right) \lambda^{n-k}[\bar{G}(x ; \xi)]^{k}
$$

and

$$
[\bar{G}(x ; \xi)]^{-(n+3 v+q-k)}=\sum_{p=0}^{\infty} \frac{\Gamma(p+n+3 v+q-k)}{\Gamma(n+3 v+q-k) p !}[G(x ; \xi)]^{p},
$$

respectively, we therefore have

$$
\begin{aligned}
\int_{0}^{\infty} f^{v}(x ; a, b, \lambda, \xi) d x & =\frac{(a b)^{v} \lambda^{2 v+n-k}(v+n)^{q}}{(1+\lambda)^{v}} \sum_{p, q, m, n=0}^{\infty} \frac{(-1)^{m+n} \Gamma((b-1) v+1)}{\Gamma((b-1) v-m) m !(1+\lambda)^{n+v}} \\
& \times \frac{\Gamma((a-1) v+a m+1)}{\Gamma((a-1) v+a m+1-n) n !} \frac{\Gamma(p+n+3 v+q-k)}{\Gamma(n+3 v+q-k) p !}\left(\begin{array}{l}
n \\
k
\end{array}\right) \\
& \times \int_{0}^{\infty} g^{v}(x ; \xi)[G(x ; \xi)]^{p+q} d x
\end{aligned}
$$

which can be written as

$$
\begin{aligned}
\int_{0}^{\infty} f^{v}(x ; a, b, \lambda, \xi) d x & =[a b]^{v} \frac{\lambda^{2 v+n-k}(v+n)^{q}}{(1+\lambda)^{v}} \sum_{p, q, m, n=0}^{\infty} \frac{(-1)^{m+n} \Gamma((b-1) v+1)}{\Gamma((b-1) v-m) m !(1+\lambda)^{n+v}} \\
& \times \frac{\Gamma((a-1) v+a m+1)}{\Gamma((a-1) v+a m+1-n) n !} \frac{\Gamma(p+n+3 v+q-k)}{\Gamma(n+3 v+q-k) p !}\left(\begin{array}{l}
n \\
k
\end{array}\right) \\
& \times\left[\frac{v}{p+q}+1\right]^{v} \int_{0}^{\infty}\left[\left(\frac{p+q}{v}+1\right) g(x ; \xi)[G(x ; \xi)]^{\frac{p+q}{v}}\right]^{v} d x . \\
& =\sum_{p, q=0}^{\infty} v_{p, q}^{*} e^{(1-v) I_{R E G}} .
\end{aligned}
$$

Consequently, Rényi entropy of KOL-G family of distributions can be expressed as

$$
I_{R}(v)=(1-v)^{-1} \log \left[\sum_{p, q=0}^{\infty} v_{p, q}^{*} e^{(1-v) I_{R E G}}\right],
$$

where $I_{R E G}=\int_{0}^{\infty}\left[\left(\frac{p+q}{v}+1\right) g(x ; \xi)[G(x ; \xi)]^{\frac{p+q}{v}}\right]^{v} d x$ represents Rényi entropy of Exp-G distribution with power parameter $\frac{p+q}{v}$ and

$$
\begin{aligned}
v_{p, q}^{*} & =\frac{(a b)^{v} \lambda^{2 v+n-k}(v+n)^{q}}{(1+\lambda)^{v}} \sum_{m, n=0}^{\infty} \frac{(-1)^{m+n} \Gamma((b-1) v+1)}{\Gamma((b-1) v-m) m !(1+\lambda)^{n+v}} \\
& \times \frac{\Gamma((a-1) v+a m+1)}{\Gamma((a-1) v+a m+1-n) n !} \frac{\Gamma(p+n+3 v+q-k)}{\Gamma(n+3 v+q-k) p !} \\
& \times\left(\begin{array}{l}
n \\
k
\end{array}\right)\left[\frac{v}{p+q}+1\right]^{v} .
\end{aligned}
$$


KOL-G distribution Rényi entropy, can be derived directly from Rényi entropy of Exp-G distribution.

\section{Maximum Likelihood Estimation}

Let $\Theta=(a, b, \lambda, \xi)^{T}$ be a $p \times 1$ parameter vector. The total $\log$-likelihood function for $\Theta$ is given by

$$
\begin{aligned}
\ell=\ell(\Theta) & =n \log (a)+n \log (b)+2 n \log (\lambda)-n \log (1+\lambda)+\sum_{i=1}^{n} \log [g(x ; \xi)] \\
& -3 \sum_{i=1}^{n} \log [1-G(x ; \xi)]-\lambda \sum_{i=1}^{n} \frac{G(x ; \xi)}{\bar{G}(x ; \xi)} \\
& +(a-1) \sum_{i=1}^{n} \log \left[1-\frac{\lambda+\bar{G}(x ; \xi)}{(1+\lambda) \bar{G}(x ; \xi)} \exp \left\{-\lambda \frac{G(x ; \xi)}{\bar{G}(x ; \xi)}\right\}\right] \\
& +(b-1) \sum_{i=1}^{n} \log \left[1-\left(1-\frac{\lambda+\bar{G}(x ; \xi)}{(1+\lambda) \bar{G}(x ; \xi)} \exp \left\{-\lambda \frac{G(x ; \xi)}{\bar{G}(x ; \xi)}\right\}\right)^{a}\right]
\end{aligned}
$$

The first derivative of the $\log$-likelihood function with respect to the parameters $\Theta=(a, b, \lambda, \xi)^{T}$, are

$$
\begin{aligned}
& \frac{\partial \ell}{\partial a}=\frac{n}{a}+\sum_{i=1}^{n} \log \left[1-\frac{\lambda+\bar{G}(x ; \xi)}{(1+\lambda) \bar{G}(x ; \xi)} \exp \left\{-\lambda \frac{G(x ; \xi)}{x ; \xi}\right\}\right]
\end{aligned}
$$

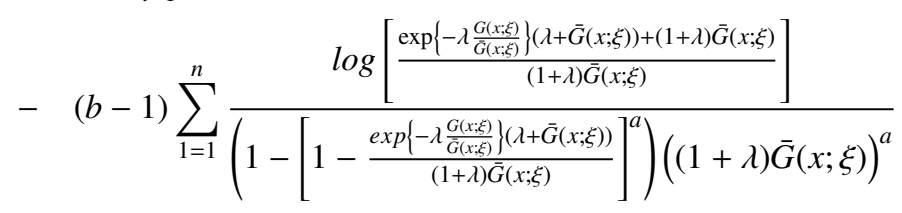

$$
\begin{aligned}
& \times\left(\exp \left\{-\lambda \frac{G(x ; \xi)}{\bar{G}(x ; \xi)}\right\}(\lambda+\bar{G}(x ; \xi))+(1+\lambda) \bar{G}(x ; \xi)\right)^{a}, \\
& \frac{\partial \ell}{\partial b}=\frac{n}{b}+\sum_{i=1}^{n} \log \left[1-\left(1-\frac{\lambda+\bar{G}(x ; \xi)}{(1+\lambda) \bar{G}(x ; \xi)} \exp \left\{-\lambda \frac{G(x ; \xi)}{\bar{G}(x ; \xi)}\right\}\right)^{a}\right] \\
& \frac{\partial \ell}{\partial \lambda}=\frac{2 n}{\lambda}-\frac{n}{(1+\lambda)}-\sum_{i=1}^{n} \frac{G(x ; \xi)}{\bar{G}(x ; \xi)}-(a-1) \\
& \times \sum_{i=1}^{n} \frac{\bar{G}(x ; \xi) \exp \left\{-\lambda \frac{G(x ; \xi)}{\bar{G}(x ; \xi)}\right\}(1-\bar{G}(x ; \xi))}{\bar{G}(x ; \xi)\left((1+\lambda) \bar{G}(x ; \xi)-\exp \left\{-\lambda \frac{G(x ; \xi)}{\bar{G}(x ; \xi)}\right\}(\lambda+\bar{G}(x ; \xi))\right)(1+\lambda)} \\
& +(a-1) \sum_{i=1}^{n} \frac{G(x ; \xi) \exp \left\{-\lambda \frac{G(x ; \xi)}{\bar{G}(x ; \xi)}\right\}(1+\lambda)(\lambda+\bar{G}(x ; \xi))}{\bar{G}(x ; \xi)\left((1+\lambda) \bar{G}(x ; \xi)-\exp \left\{-\lambda \frac{G(x ; \xi)}{\bar{G}(x ; \xi)}\right\}(\lambda+\bar{G}(x ; \xi))\right)(1+\lambda)} \\
& +(b-1) \\
& \times \sum_{i=1}^{n} \frac{a\left(\bar{G}(x ; \xi) \exp \left\{-\lambda \frac{G(x ; \xi)}{\bar{G}(x ; \xi)}\right\}-G(x ; \xi) \exp \left\{-\lambda \frac{G(x ; \xi)}{\bar{G}(x ; \xi)}\right\}(1+\lambda)(\lambda+\bar{G}(x ; \xi))\right)}{G^{\bar{a}+1}(x ; \xi)\left[1-\left(1-\frac{\lambda+\bar{G}(x ; \xi)}{(1+\lambda) \bar{G}(x ; \xi)} \exp \left\{-\lambda \frac{G(x ; \xi)}{\bar{G}(x ; \xi)}\right\}\right)^{a}\right](1+\lambda)^{a+1}} \\
& \times\left(\bar{G}(x ; \xi)(1+\lambda)-\exp \left\{-\lambda \frac{G(x ; \xi)}{\bar{G}(x ; \xi)}\right\}(\lambda+\bar{G}(x ; \xi))\right)^{a-1},
\end{aligned}
$$


and

$$
\begin{aligned}
\frac{\partial \ell}{\partial \xi_{k}} & =\sum_{i=1}^{n} \frac{1}{g(x ; \xi)} \frac{\partial g(x ; \xi)}{\partial \xi_{k}}+3 \sum_{i=0}^{n} \frac{1}{\bar{G}(x ; \xi)} \frac{\partial G(x ; \xi)}{\partial \xi_{k}}-\lambda \sum_{i=1}^{n} \frac{G(x ; \xi) \frac{\partial G(x ; \xi)}{\partial \xi_{k}}-\bar{G}(x ; \xi) \frac{\partial G(x ; \xi)}{\partial \xi_{k}}}{[\bar{G}(x ; \xi)]^{2}} \\
& +(a-1) \sum_{i=1}^{n} \frac{1}{\left[1-\frac{\lambda+\bar{G}(x ; \xi)}{(1+\lambda) \bar{G}(x ; \xi)} \exp \left\{-\lambda \frac{G(x ; \xi)}{\bar{G}(x ; \xi)}\right]\right.} \frac{\partial\left[1-\frac{\lambda+\bar{G}(x ; \xi)}{(1+\lambda) \bar{G}(x ; \xi)} \exp \{-\lambda \overline{G(x ; \xi)})\right]}{\partial \xi_{k}} \\
& +(b-1) \sum_{i=1}^{n} \frac{1}{\left[1-\left(1-\frac{\lambda+\bar{G}(x ; \xi)}{(1+\lambda) \bar{G}(x ; \xi)} \exp \left\{-\lambda \frac{G(x ; \xi)}{\bar{G}(x ; \xi)}\right)\right)^{a}\right]} \\
& \times \frac{\partial\left[1-\left(1-\frac{\lambda+\bar{G}(x ; \xi)}{(1+\lambda) \bar{G}(x ; \xi)} \exp \left\{-\lambda \frac{G(x ; \xi)}{\partial \bar{G}(x ; \xi)}\right)\right)^{a}\right]}{\partial \xi_{k}} .
\end{aligned}
$$

\section{Special Cases}

Some cases which includes Kumaraswamy Odd Lindley-uniform (KOL-U), Kumaraswamy Odd Lindley-log-logistic (KOL-LLo), and Kumaraswamy Odd Lindley-Weibull (KOL-W) distributions are presented. Statistical properties, distribution of order statistics and Rényi entropy of these cases are also studied.

\subsection{Kumaraswamy-Odd-Lindley-Uniform (KOL-U) Distribution}

Suppose the baseline distribution is a uniform distribution on the interval $(0, \theta)$ with $\theta>0$. The baseline pdf and cdf are $g(x ; \theta)=1 / \theta$ and $G(x ;, \theta)=x / \theta$, respectively. The KOL-U distribution have cdf and pdf given by

$$
F_{K O L-U}(x ; a, b, \lambda, \theta)=1-\left(1-\left[1-\frac{\lambda+(1-x / \theta)}{(1+\lambda)(1-x / \theta)} \exp \left\{-\lambda \frac{x}{(x-\theta)}\right\}\right]^{a}\right)^{b}
$$

and

$$
\begin{aligned}
f_{K O L-U}(x) & =a b\left[\frac{\lambda^{2}}{(1+\lambda)} \frac{(1 / \theta)}{(1-x / \theta)^{3}} \exp \left\{-\lambda \frac{x}{(\theta-x)}\right\}\right] \\
& \times\left[1-\frac{\lambda+(1-x / \theta)}{(1+\lambda)(1-x / \theta)} \exp \left\{-\lambda \frac{x}{(\theta-x)}\right\}\right]^{a-1} \\
& \times\left(1-\left[1-\frac{\lambda+(1-x / \theta)}{(1+\lambda)(1-x / \theta)} \exp \left\{-\lambda \frac{x}{(\theta-x)}\right\}\right]^{a}\right)^{b-1},
\end{aligned}
$$

respectively. Graphs of the KOL-U pdf take various shapes including platykurtic, left skewed and unimodal.

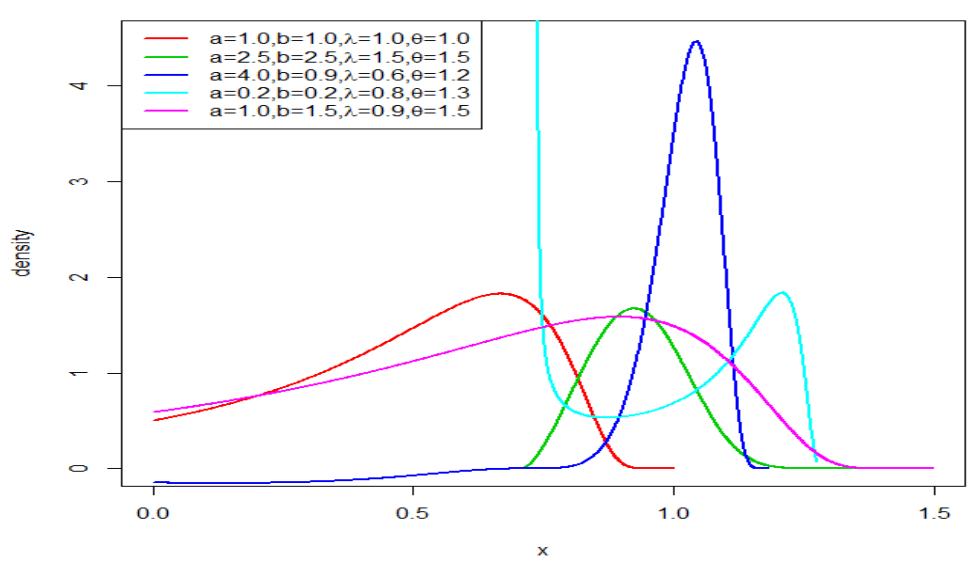

Figure 1. Plots of KOL-U pdf 


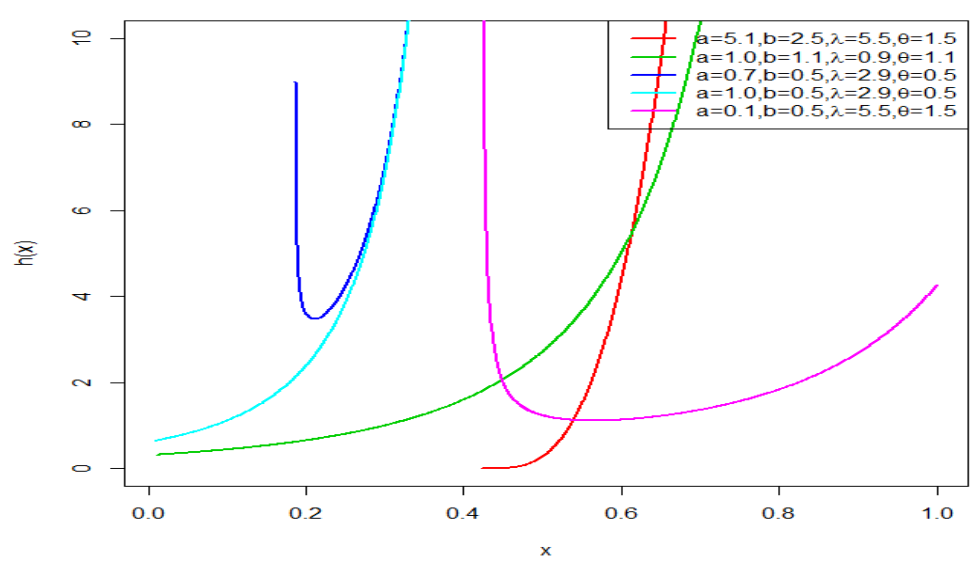

Figure 2. Plots of KOL-U Hazard Rate Function

\subsubsection{Nested Models}

Some of the sub-models of the KOL-U distribution are presented below.

- We obtain Odd Lindley-Uniform (OL-U) distribution from KOL-U distribution by setting $a=b=1$.

- We obtain a power function distribution denoted by $K O L-U(1, b, \lambda, \theta)$ from KOL-U distribution by setting $a=1$, with pdf given by

$$
f(x)=b\left[\frac{\lambda^{2}}{(1+\lambda)} \frac{(1 / \theta)}{(1-x / \theta)^{3}} e^{\left\{-\lambda \frac{x}{(\theta-x)}\right\}}\right]\left(\frac{\lambda+(1-x / \theta)}{(1+\lambda)(1-x / \theta)} e^{\left\{-\lambda \frac{x}{(\theta-x)}\right\}}\right)^{b-1} .
$$

- The exponentiated-Odd Lindley-U (EOL-U) distribution denoted by $K O L-U(a, 1, \lambda, \theta)$ is obtained from the $\mathrm{KOL}$ $\mathrm{U}$ distribution by setting $b=1$, with pdf given by

$$
f(x)=a\left[\frac{\lambda^{2}}{(1+\lambda)} \frac{(1 / \theta)}{(1-x / \theta)^{3}} e^{\left\{-\lambda \frac{x}{(\theta-x)}\right\}}\right]\left[1-\frac{\lambda+(1-x / \theta)}{(1+\lambda)(1-x / \theta)} e^{\left\{-\lambda \frac{x}{(\theta-x)}\right\}}\right]^{a-1} .
$$

\subsubsection{Hazard Rate and Quantile Functions}

Hazard rate of KOL-U distribution is computed by the following formula

$$
h_{K O L-U}(x)=\frac{f_{K O L-U}(x)}{\bar{F}_{K O L-U}(x)},
$$

where $f_{K O L-U}(x)$ is given by equation (23), $\bar{F}_{K O L-U}(x)=1-F_{K O L-U}(x)$ and $F_{K O L-U}(x)$ is the KOL-U cdf as in equation (22). Hazard function for KOL-U distribution can take various shapes including bathtub and increasing for the selected parameter values.

We obtain the quantile function of KOL-U from equation (11) and is given by

$$
Q(u)=\theta\left(1+\lambda\left[1+W_{-}\left(\left[\left((u-1)^{1 / b}+1\right)^{1 / a}+1\right](1+\lambda) \exp (-(1+\lambda))\right)\right]^{-1}\right) .
$$

\subsection{Kumaraswamy Odd Lindley-Log-Logistic (KOL-LLo) Distribution}

Suppose that the baseline distribution is a log-logistic distribution on the interval $(0, \infty)$, where $g(x)=c x^{c-1}\left(1+x^{c}\right)^{-2}$ and $G(x)=1-\left(1+x^{c}\right)^{-1}$. The cdf and pdf of the KOL-LLo distribution are given by

$$
F_{K O L-L L o}(x)=1-\left(1-\left[1-\frac{\lambda+\left(\left(1+x^{c}\right)^{-1}\right)}{(1+\lambda)\left(\left(1+x^{c}\right)^{-1}\right)} \exp \left\{-\lambda \frac{\left(1-\left(1+x^{c}\right)^{-1}\right)}{\left(\left(1+x^{c}\right)^{-1}\right)}\right\}\right]^{a}\right)^{b},
$$




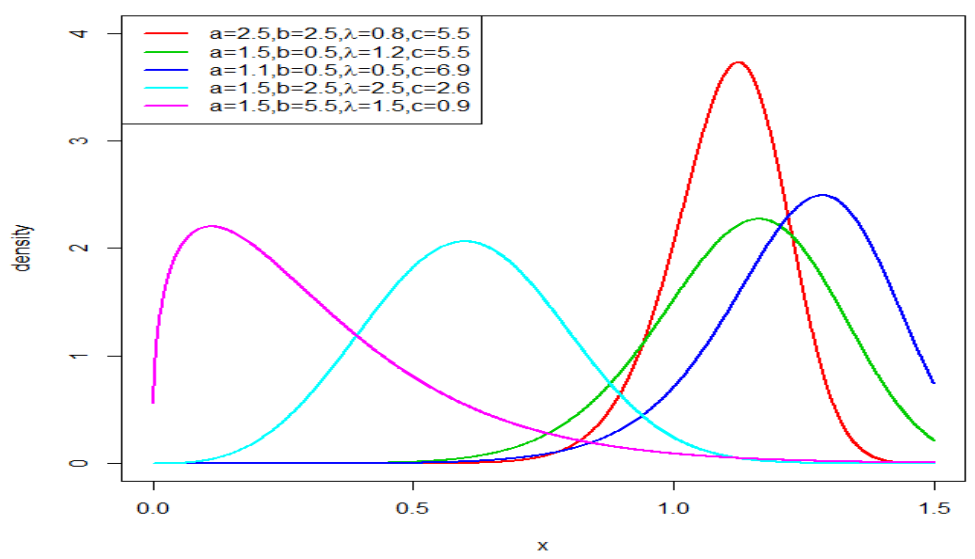

Figure 3. Plots of KOL-LLo Density Function

and

$$
\begin{aligned}
f_{K O L-L L o}(x) & =a b\left[\frac{\lambda^{2}}{(1+\lambda)} \frac{c x^{c-1}}{\left(1+x^{c}\right)^{-1}} \exp \left\{-\lambda \frac{1-\left(1+x^{c}\right)^{-1}}{\left(\left(1+x^{c}\right)^{-1}\right)}\right\}\right] \\
& \times\left[1-\frac{\lambda+\left(\left(1+x^{c}\right)^{-1}\right)}{(1+\lambda)\left(\left(1+x^{c}\right)^{-1}\right)} \exp \left\{-\lambda \frac{\left(1-\left(1+x^{c}\right)^{-1}\right)}{\left(\left(1+x^{c}\right)^{-1}\right)}\right\}\right]^{a-1} \\
& \times\left(1-\left[1-\frac{\lambda+\left(\left(1+x^{c}\right)^{-1}\right)}{(1+\lambda)\left(\left(1+x^{c}\right)^{-1}\right)} \exp \left\{-\lambda \frac{\left(1-\left(1+x^{c}\right)^{-1}\right)}{\left(\left(1+x^{c}\right)^{-1}\right)}\right\}\right]^{a}\right)^{b-1},
\end{aligned}
$$

respectively.

KOL-LLo density function takes various shapes including right skewed, left skewed and symmetric.

\subsubsection{Nested Models}

- We obtain odd Lindley-LLo distribution from KOL-LLo distribution by setting $a=b=1$.

- We also obtain a power function distribution from KOL-LLo distribution by setting $a=1$. This sub-model is denoted by $K O L-L L o(1, b, \lambda, c)$, and the pdf

$$
\begin{aligned}
f(x) & =b\left[\frac{\lambda^{2}}{(1+\lambda)} \frac{c x^{c-1}}{\left(1+x^{c}\right)^{-1}} \exp \left\{-\lambda \frac{1-\left(1+x^{c}\right)^{-1}}{\left(\left(1+x^{c}\right)^{-1}\right)}\right\}\right] \\
& \times\left(\frac{\lambda+\left(\left(1+x^{c}\right)^{-1}\right)}{(1+\lambda)\left(\left(1+x^{c}\right)^{-1}\right)} \exp \left\{-\lambda \frac{\left(1-\left(1+x^{c}\right)^{-1}\right)}{\left(\left(1+x^{c}\right)^{-1}\right)}\right\}\right)^{b-1} .
\end{aligned}
$$

- The exponentiated odd Lindley-Log-logistic, denoted by $(K O L-L L o(a, 1, \lambda, c)$ or EOL-LLo) is obtained by setting $b=1$, with pdf

$$
\begin{aligned}
f(x) & =a\left[\frac{\lambda^{2}}{(1+\lambda)} \frac{c x^{c-1}}{\left(1+x^{c}\right)^{-1}} \exp \left\{-\lambda \frac{1-\left(1+x^{c}\right)^{-1}}{\left(\left(1+x^{c}\right)^{-1}\right)}\right\}\right] \\
& \times\left[1-\frac{\lambda+\left(\left(1+x^{c}\right)^{-1}\right)}{(1+\lambda)\left(\left(1+x^{c}\right)^{-1}\right)} \exp \left\{-\lambda \frac{G(x ; \xi)}{\left(\left(1+x^{c}\right)^{-1}\right)}\right\}\right]^{a-1} .
\end{aligned}
$$

- When $a=b=c=1$, the KOL-LLo density function reduces a one parameter KOL-LLo distribution denoted by $K O L-L L o(1,1, \lambda, 1)$ and with the pdf

$$
f(x)=\left[\frac{\lambda^{2}}{(1+\lambda)} \frac{1}{(1+x)^{-1}} \exp \left\{-\lambda \frac{1-(1+x)^{-1}}{\left((1+x)^{-1}\right)}\right\}\right] .
$$


- Also, when $a=c=1$, the KOL-LLo distribution becomes a two parameter KOL-LLo distribution denoted by $K O L-L L o(1, b, \lambda, 1)$ distribution with pdf

$$
\begin{aligned}
f(x) & =b\left[\frac{\lambda^{2}}{(1+\lambda)} \frac{1}{(1+x)^{-1}} \exp \left\{-\lambda \frac{1-(1+x)^{-1}}{\left((1+x)^{-1}\right)}\right\}\right] \\
& \times\left(\frac{\lambda+\left((1+x)^{-1}\right)}{(1+\lambda)\left((1+x)^{-1}\right)} \exp \left\{-\lambda \frac{\left(1-(1+x)^{-1}\right)}{\left((1+x)^{-1}\right)}\right\}\right)^{b-1} .
\end{aligned}
$$

- When $b=c=1$, the KOL-LLo distribution becomes a two parameter KOL-LLo distribution denoted by KOL $\operatorname{LLo}(a, 1, \lambda, 1)$ distribution with pdf

$$
\begin{aligned}
f(x) & =a\left[\frac{\lambda^{2}}{(1+\lambda)} \frac{1}{(1+x)^{-1}} \exp \left\{-\lambda \frac{1-(1+x)^{-1}}{\left((1+x)^{-1}\right)}\right\}\right] \\
& \times\left[1-\frac{\lambda+\left((1+x)^{-1}\right)}{(1+\lambda)\left((1+x)^{-1}\right)} \exp \left\{-\lambda \frac{\left(1-(1+x)^{-1}\right)}{\left((1+x)^{-1}\right)}\right\}\right]^{a-1}
\end{aligned}
$$

\subsubsection{Hazard Rate and Quantile Functions}

The hazard function for KOL-LLo distribution is defined by

$$
h_{K O L-L L o}(x)=\frac{f_{K O L-L L o}(x)}{\bar{F}_{K O L-L L o}(x)},
$$

where $f_{K O L-L L o}(x)$ is given by equation $(26), \bar{F}_{K O L-L L o}(x)=1-F_{K O L-L L o}(x)$ and $F_{K O L-L L o}(x)$ is the KOL-LLo cdf as in equation (25).

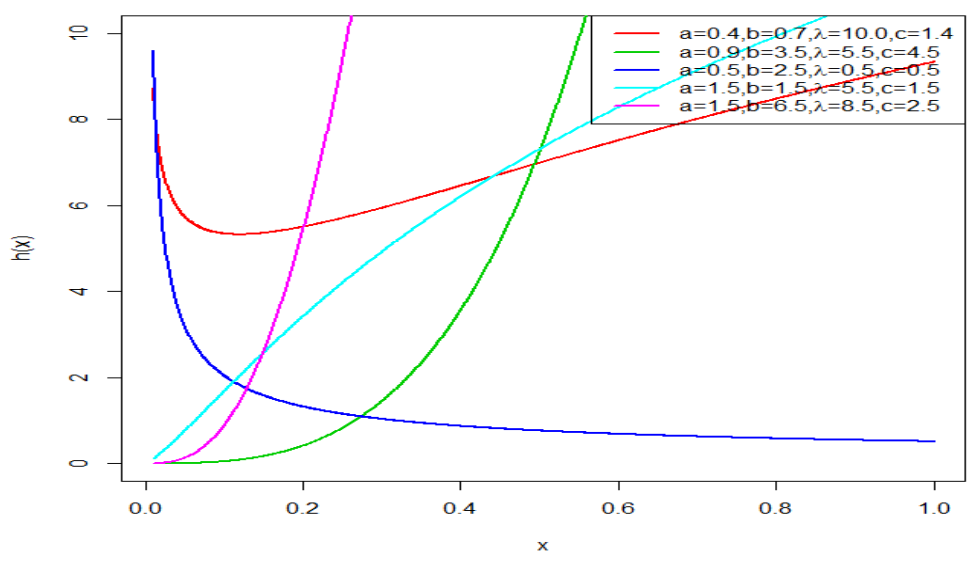

Figure 4. Plots of KOL-LLo Hazard Rate Function

Hazard function for KOL-U distribution can take various shapes including bathtub, decreasing and increasing for the selected parameter values. We obtain the quantile function of KOL-LLo distribution from equation (11), and is given by

$$
Q(u)=\left[\left(1-\left(1+\lambda\left[1+W_{-}\left(\left[\left((u-1)^{1 / b}+1\right)^{1 / a}+1\right](1+\lambda) e^{-(1+\lambda)}\right)\right]^{-1}\right)\right)^{-1}-1\right]^{1 / c}
$$

\subsubsection{Moments}

Let $X \sim K O L-L L o(a, b, \lambda ; c)$, the $s^{\text {th }}$ moment of KOL-LLo distribution comes directly from equation (6), and is given by

$$
E\left(X^{s}\right)=\sum_{p, q, m, n=0}^{\infty} v_{p, q, m, n} E\left(W_{p+q}^{s}\right)
$$


Table 1. Table of Quantiles for Selected Parameters of KOL-LLo Distribution

\begin{tabular}{lccccc}
\hline $\mathrm{u}$ & $(0.6,1.5,1,0.5)$ & $(0.9,1.4,1.5,0.9)$ & $(0.5,0.8,2,0.6)$ & $(1.1,1.5,1.5,0.2)$ & $(3,2.5,0.5,0.95)$ \\
\hline 0.1 & 0.00048922 & 0.0448213 & 0.0006011 & 0.00010855 & 1.8341880 \\
0.2 & 0.00547633 & 0.1101525 & 0.0057784 & 0.00029428 & 2.4014390 \\
0.3 & 0.02276702 & 0.1911443 & 0.0223695 & 0.00223242 & 2.8654280 \\
0.4 & 0.06475537 & 0.2894192 & 0.0593949 & 0.01092732 & 3.2992610 \\
0.5 & 0.15178761 & 0.4093259 & 0.1301308 & 0.04197372 & 3.7368240 \\
0.6 & 0.31967522 & 0.5591120 & 0.2562518 & 0.14258116 & 4.2064230 \\
0.7 & 0.64139131 & 0.7549405 & 0.4792034 & 0.46932070 & 4.7457330 \\
0.8 & 1.29897513 & 1.0335419 & 0.8963855 & 1.65814419 & 5.4276690 \\
0.9 & 2.96467690 & 1.5128419 & 1.8446854 & 7.84523292 & 6.4699320 \\
\hline
\end{tabular}

where $E\left(W_{p+q}^{s}\right)$ denotes the $s^{\text {th }}$ moment of $W_{p+q}$ which follows an E-LLo distribution with power parameter $(p+q)$ and $v_{p, q, m, n}$ is as defined in equation (7).

We present some results on the first six moments and the measures of dispersion for the KOL-LLo distribution for some parameter values. In the first set of results, we fixed the parameters $a=2.0$ and $b=1.0$ and fixing the parameters $\lambda=1.5$ and $c=1.5$ in the second set of results.

Table 2. Moments of the KOLL-Lo distribution for some parameter values (fixing $a=2.0$ and $b=1.0$ and fixing $\lambda=1.5$ and $c=1.5)$

\begin{tabular}{lcccc|cccc}
\hline & $\lambda=3.5, \mathrm{c}=1.5$ & $\lambda=3.5, \mathrm{c}=0.8$ & $\lambda=1.5, \mathrm{c}=1.5$ & $\lambda=0.8, \mathrm{c}=3.5$ & $\mathrm{a}=1.0, \mathrm{~b}=1.5$ & $\mathrm{a}=2.5, \mathrm{~b}=2.5$ & $\mathrm{a}=1.0, \mathrm{~b}=1.0$ & $\mathrm{a}=1.5, \mathrm{~b}=1.5$ \\
\hline $\mathrm{E}(\mathrm{X})$ & 0.6126209 & 0.4742228 & 1.1762579 & 1.2885425 & 0.6801175 & 0.9059092 & 0.8726366 & 0.8590534 \\
$E\left(X^{2}\right)$ & 0.4622079 & 0.4085050 & 1.6702770 & 1.7216853 & 0.6490846 & 0.9338809 & 1.0632330 & 0.9288587 \\
$E\left(X^{3}\right)$ & 0.4096750 & 0.5312524 & 2.7466667 & 2.3750889 & 0.7664072 & 1.0684230 & 1.6000000 & 1.1843770 \\
$E\left(X^{4}\right)$ & 0.4142154 & 0.9469599 & 5.0980449 & 3.3716287 & 1.0572909 & 1.3347006 & 2.8123959 & 1.7188370 \\
$E\left(X^{5}\right)$ & 0.4681821 & 2.1776613 & 10.4936256 & 4.9124491 & 1.6495011 & 1.7999642 & 5.5929596 & 2.7763381 \\
$E\left(X^{6}\right)$ & 0.5828436 & 6.1962091 & 23.6444444 & 7.3304795 & 2.8496868 & 2.5984323 & 12.3259259 & 4.9146552 \\
$\mathrm{SD}$ & 0.2947941 & 0.4285064 & 0.5354385 & 0.2476764 & 0.4318851 & 0.3364661 & 0.5493073 & 0.4369050 \\
$\mathrm{CV}$ & 0.4812016 & 0.9035971 & 0.4552051 & 0.1922144 & 0.6350154 & 0.3714126 & 0.6294800 & 0.5085888 \\
$\mathrm{CS}$ & 0.7821967 & 2.0764582 & 0.7005340 & -0.0963096 & 0.8842893 & 0.4540892 & 0.8782530 & 0.7011530 \\
$\mathrm{CK}$ & 3.7823849 & 10.0462648 & 3.6229853 & 3.0104233 & 3.7900378 & 3.2048982 & 3.7978240 & 3.5152632 \\
\hline
\end{tabular}

\subsection{Kumaraswamy Odd Lindley-Weibull (KOL-W) Distribution}

Suppose the baseline distribution is Weibull distribution with parameters $c$ and $k$, with pdf and cdf given by $g(x ; c, k)=$ $\frac{k}{c}\left(\frac{x}{c}\right)^{k-1} e^{-\left(\frac{x}{c}\right)^{k}}$ and $G(x ; c, k)=1-e^{-\left(\frac{x}{c}\right)^{k}}$, respectively, then the cdf and pdf of KOL-W distribution are given by

$$
F_{K O L-W}(x)=1-\left(1-\left[1-\frac{\lambda+\left(e^{-\left(\frac{x}{c}\right)^{k}}\right)}{(1+\lambda)\left(e^{-\left(\frac{x}{c}\right)^{k}}\right)} \exp \left\{-\lambda \frac{\left(1-e^{-\left(\frac{x}{c}\right)^{k}}\right)}{\left(e^{-\left(\frac{x}{c}\right)^{k}}\right)}\right\}\right]^{a}\right)^{b}
$$

and

$$
\begin{aligned}
& f_{K O L-W}(x)=a b\left[\frac{\lambda^{2}}{1+\lambda} \frac{\frac{k}{c}\left(\frac{x}{c}\right)^{k-1}}{\left(e^{-\left(\frac{x}{c}\right)^{k}}\right)^{2}} \exp \left\{-\lambda \frac{\left(1-e^{-\left(\frac{x}{c}\right)^{k}}\right)}{\left(e^{-\left(\frac{x}{c}\right)^{k}}\right)}\right\}\right] \\
& \times\left[1-\frac{\lambda+\left(e^{-\left(\frac{x}{c}\right)^{k}}\right)}{(1+\lambda)\left(e^{-\left(\frac{x}{c}\right)^{k}}\right)} \exp \left\{-\lambda \frac{\left(1-e^{-\left(\frac{x}{c}\right)^{k}}\right)}{\left(e^{-\left(\frac{x}{c}\right)^{k}}\right)}\right\}\right]^{a-1} \\
& \times\left(1-\left[1-\frac{\lambda+\left(e^{-\left(\frac{x}{c}\right)^{k}}\right)}{(1+\lambda)\left(e^{-\left(\frac{x}{c}\right)^{k}}\right)} \exp \left\{-\lambda \frac{\left(1-e^{-\left(\frac{x}{c}\right)^{k}}\right)}{\left(e^{-\left(\frac{x}{c}\right)^{k}}\right)}\right\}\right]^{a}\right)^{b-1},
\end{aligned}
$$

respectively. Plots of the KOL-W density function takes various shapes including decreasing, unimodal, left and right skewed for the selected parameter values. 


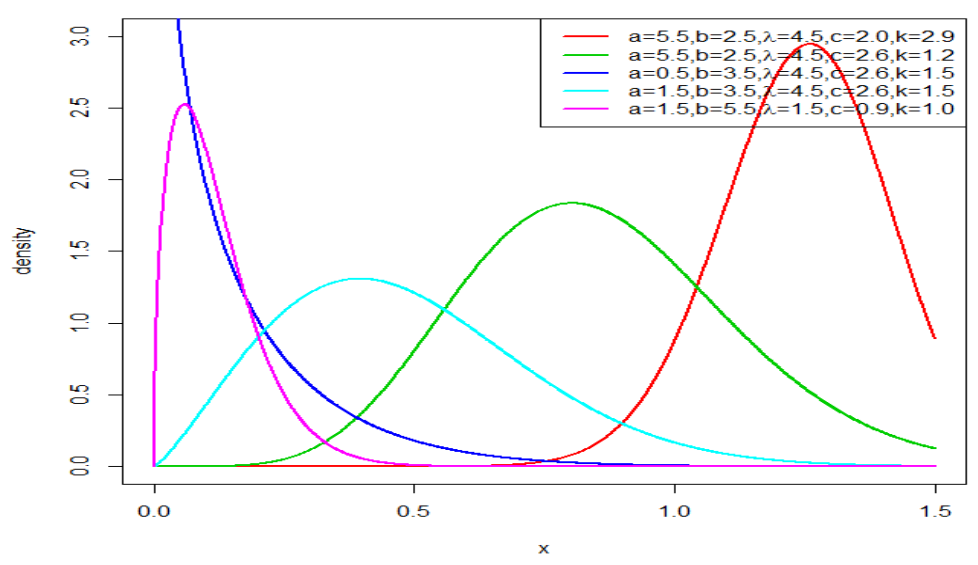

Figure 5. Plots of KOL-W Density Function

\subsubsection{Nested Models}

- We obtain the Odd Lindley-Weibull (OL-W) distribution from KOL-W distribution by setting $a=b=1$.

- We also obtain a power function distribution from KOL-W distribution by setting $a=1$, given by

$$
\begin{aligned}
f(x) & =b\left[\frac{\lambda^{2}}{1+\lambda} \frac{\frac{k}{c}\left(\frac{x}{c}\right)^{k-1}}{\left(e^{-\left(\frac{x}{c}\right)^{k}}\right)^{2}} \exp \left\{-\lambda \frac{\left(1-e^{-\left(\frac{x}{c}\right)^{k}}\right)}{\left(e^{-\left(\frac{x}{c}\right)^{k}}\right)}\right\}\right] \\
& \times\left(\frac{\lambda+\left(e^{-\left(\frac{x}{c}\right)^{k}}\right)}{(1+\lambda)\left(e^{-\left(\frac{x}{c}\right)^{k}}\right)} \exp \left\{-\lambda \frac{\left(1-e^{-\left(\frac{x}{c}\right)^{k}}\right)}{\left(e^{-\left(\frac{x}{c}\right)^{k}}\right)}\right\}\right)^{b-1} .
\end{aligned}
$$

- The exponentiated-odd Lindley-Weibull (EOL-W) distribution is obtained by setting $b=1$, with pdf given by

$$
\begin{aligned}
f(x) & =a\left[\frac{\lambda^{2}}{1+\lambda} \frac{\frac{k}{c}\left(\frac{x}{c}\right)^{k-1}}{\left(e^{-\left(\frac{x}{c}\right)^{k}}\right)^{2}} \exp \left\{-\lambda \frac{\left(1-e^{-\left(\frac{x}{c}\right)^{k}}\right)}{\left(e^{-\left(\frac{x}{c}\right)^{k}}\right)}\right\}\right] \\
& \times\left[1-\frac{\lambda+\left(e^{-\left(\frac{x}{c}\right)^{k}}\right)}{(1+\lambda)\left(e^{-\left(\frac{x}{c}\right)^{k}}\right)} \exp \left\{-\lambda \frac{\left(1-e^{-\left(\frac{x}{c}\right)^{k}}\right)}{\left(e^{-\left(\frac{x}{c}\right)^{k}}\right)}\right\}\right]^{a-1} .
\end{aligned}
$$

- By setting $k=1$ and $k=2$, we obtain the Kumaraswamy odd Lindley- exponential (KOL-E) distribution and Kumaraswamy odd Lindley-Rayleigh (KOL-R) distribution with pdfs given by

$$
\begin{aligned}
f(x) & =a b\left[\frac{\lambda^{2}}{1+\lambda} \frac{\left(\frac{1}{c}\right)}{\left(e^{-\left(\frac{x}{c}\right)}\right)^{2}} \exp \left\{-\lambda \frac{\left(1-e^{-\left(\frac{x}{c}\right)}\right)}{\left(e^{-\left(\frac{x}{c}\right)}\right)}\right\}\right] \\
& \times\left[1-\frac{\lambda+\left(e^{-\left(\frac{x}{c}\right)}\right)}{(1+\lambda)\left(e^{-\left(\frac{x}{c}\right)}\right)} \exp \left\{-\lambda \frac{\left(1-e^{-\left(\frac{x}{c}\right)}\right)}{\left(e^{-\left(\frac{x}{c}\right)}\right)}\right\}\right]^{a-1} \\
& \times\left(1-\left[1-\frac{\lambda+\left(e^{-\left(\frac{x}{c}\right)}\right)}{(1+\lambda)\left(e^{-\left(\frac{x}{c}\right)}\right)} \exp \left\{-\lambda \frac{\left(1-e^{-\left(\frac{x}{c}\right)}\right)}{\left(e^{-\left(\frac{x}{c}\right)}\right)}\right\}\right]^{a}\right)^{b-1},
\end{aligned}
$$


and

$$
\begin{aligned}
& f(x)=a b\left[\frac{\lambda^{2}}{1+\lambda} \frac{\frac{2}{c}\left(\frac{x}{c}\right)}{\left(e^{-\left(\frac{x}{c}\right)^{2}}\right)^{2}} \exp \left\{-\lambda \frac{\left(1-e^{-\left(\frac{x}{c}\right)^{2}}\right)}{\left(e^{-\left(\frac{x}{c}\right)^{2}}\right)}\right\}\right] \\
& \times\left[1-\frac{\lambda+\left(e^{-\left(\frac{x}{c}\right)^{2}}\right)}{(1+\lambda)\left(e^{-\left(\frac{x}{c}\right)^{2}}\right)} \exp \left\{-\lambda \frac{\left(1-e^{-\left(\frac{x}{c}\right)^{2}}\right)}{\left(e^{-\left(\frac{x}{c}\right)^{2}}\right)}\right\}\right]^{a-1} \\
& \times\left(1-\left[1-\frac{\lambda+\left(e^{-\left(\frac{x}{c}\right)^{2}}\right)}{(1+\lambda)\left(e^{-\left(\frac{x}{c}\right)^{2}}\right)} \exp \left\{-\lambda \frac{\left(1-e^{-\left(\frac{x}{c}\right)^{2}}\right)}{\left(e^{-\left(\frac{x}{c}\right)^{2}}\right)}\right\}\right]^{a}\right)^{b-1},
\end{aligned}
$$

respectively.

- Also, when $a=k=1$ and $b=k=1$, we obtain the pdfs

$$
\begin{aligned}
f(x) & =b\left[\frac{\lambda^{2}}{1+\lambda} \frac{\left(\frac{1}{c}\right)}{\left(e^{-\left(\frac{x}{c}\right)}\right)^{2}} \exp \left\{-\lambda \frac{\left(1-e^{-\left(\frac{x}{c}\right)}\right)}{\left(e^{-\left(\frac{x}{c}\right)}\right)}\right\}\right] \\
& \times\left(\frac{\lambda+\left(e^{-\left(\frac{x}{c}\right)}\right)}{(1+\lambda)\left(e^{-\left(\frac{x}{c}\right)}\right)} \exp \left\{-\lambda \frac{\left(1-e^{-\left(\frac{x}{c}\right)}\right)}{\left(e^{-\left(\frac{x}{c}\right)}\right)}\right\}\right)^{b-1} .
\end{aligned}
$$

and

$$
\begin{aligned}
f(x) & =a\left[\frac{\lambda^{2}}{1+\lambda} \frac{\left(\frac{1}{c}\right)}{\left(e^{-\left(\frac{x}{c}\right)^{k}}\right)^{2}} \exp \left\{-\lambda \frac{\left(1-e^{-\left(\frac{x}{c}\right)}\right)}{\left(e^{-\left(\frac{x}{c}\right)}\right)}\right\}\right] \\
& \times\left[1-\frac{\lambda+\left(e^{-\left(\frac{x}{c}\right)}\right)}{(1+\lambda)\left(e^{-\left(\frac{x}{c}\right)}\right)} \exp \left\{-\lambda \frac{\left(1-e^{-\left(\frac{x}{c}\right)}\right)}{\left(e^{-\left(\frac{x}{c}\right)}\right)}\right\}\right]^{a-1},
\end{aligned}
$$

which can be denoted by $K O L-W(1, b, \lambda, 1, c)$ and $K O L-W(a, 1, \lambda, 1, c)$, respectively.

- By setting $a=1, k=2$ and $b=1, k=2$, we obtain the distributions with pdfs

$$
\begin{aligned}
f(x) & =b\left[\frac{\lambda^{2}}{1+\lambda} \frac{\left(\frac{2}{c}\right)\left(\frac{x}{c}\right)}{\left(e^{-\left(\frac{x}{c}\right)^{2}}\right)^{2}} \exp \left\{-\lambda \frac{\left(1-e^{-\left(\frac{x}{c}\right)^{2}}\right)}{\left(e^{-\left(\frac{x}{c}\right)^{2}}\right)}\right\}\right] \\
& \times\left(\frac{\lambda+\left(e^{-\left(\frac{x}{c}\right)^{2}}\right)}{(1+\lambda)\left(e^{-\left(\frac{x}{c}\right)^{2}}\right)} \exp \left\{-\lambda \frac{\left(1-e^{-\left(\frac{x}{c}\right)^{2}}\right)}{\left(e^{-\left(\frac{x}{c}\right)^{2}}\right)}\right\}\right)^{b-1}
\end{aligned}
$$

and

$$
\begin{aligned}
f(x) & =a\left[\frac{\lambda^{2}}{1+\lambda} \frac{\left(\frac{2}{c}\right)\left(\frac{x}{c}\right)}{\left(e^{-\left(\frac{x}{c}\right)^{2}}\right)^{2}} \exp \left\{-\lambda \frac{\left(1-e^{-\left(\frac{x}{c}\right)^{2}}\right)}{\left(e^{-\left(\frac{x}{c}\right)^{2}}\right)}\right\}\right] \\
& \times\left[1-\frac{\lambda+\left(e^{-\left(\frac{x}{c}\right)^{2}}\right)}{(1+\lambda)\left(e^{-\left(\frac{x}{c}\right)^{2}}\right)} \exp \left\{-\lambda \frac{\left(1-e^{-\left(\frac{x}{c}\right)^{2}}\right)}{\left(e^{-\left(\frac{x}{c}\right)^{2}}\right)}\right\}\right]^{a-1},
\end{aligned}
$$

which are denoted by $K O L-W(1, b, \lambda, 2, c)$ and $K O L-W(a, 1, \lambda, 2, c)$, respectively.

\subsubsection{Hazard Rate and Quantile Functions}

The hazard rate function of the KOL-W distribution was obtained by dividing KOL-W density function by its survival function

$$
h_{K O L-W}(x)=\frac{f_{K O L-W}(x)}{\bar{F}_{K O L-W}(x)},
$$

where $f_{K O L-W}(x)$ is given by equation (28), $\bar{F}_{K O L-W}(x)=1-F_{K O L-W}(x)$ and $F_{K O L-W}(x)$ is the KOL-W cdf as in equation (27). Hazard function for KOL-W distribution can take various shapes including bathtub, decreasing and increasing for the selected parameter values. We obtain the quantile function of KOL-W distribution from equation (11), and is given by

$$
Q(u)=-\frac{c}{k} \log \left[1-\left(1+\lambda\left[1+W_{-}\left(\left[\left((u-1)^{1 / b}+1\right)^{1 / a}+1\right](1+\lambda) e^{-(1+\lambda)}\right)\right]^{-1}\right)\right] .
$$




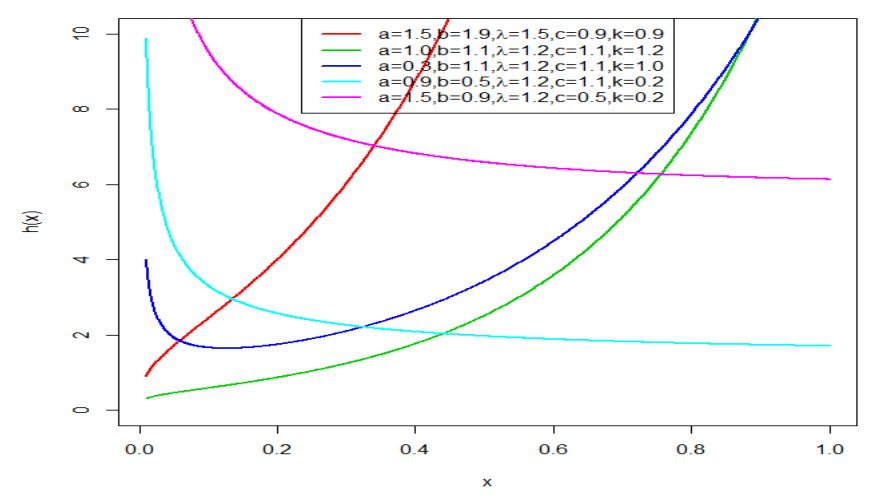

Figure 6. Plots of KOL-W Hazard Rate Function

\section{Simulation Study}

A simulation study for the KOL-LLo distribution was conducted. The simulation study was repeated for $\mathrm{N}=1000$ times with sample size $\mathrm{n}=25,50,100,200,400$ and 800 . The following parameter values were used in the simulation study: $a=1.0, b=1.0, \lambda=0.5, c=0.5$ and $a=1.5, b=1.0, \lambda=1.5, c=0.5$. The average bias (ABias) and Root Mean Square Errors (RMSEs) were computed and are given by:

$$
\operatorname{ABias}(\hat{\theta})=\sum_{i=1}^{N} \frac{\hat{\theta}_{i}}{N}-\theta \quad \text { and } \quad \operatorname{RMSE}(\hat{\theta})=\sqrt{\frac{\sum_{i=1}^{N}\left(\hat{\theta}_{i}-\theta\right)^{2}}{N}},
$$

respectively. Tables 6.1 and 6.2 presents the mean MLEs of the model parameters along with the respective average bias and root mean square errors (RMSE) for the KOL-LLo distribution for selected parameter values. Note that from the tabulated results, we can verify that as the sample size increases, the mean estimates of the parameters tend to be closer to the true parameter values, since average bias and RMSEs decays toward zero for all the parameters.

\section{Application}

In this section, we present an application of the KOL-LLo distribution to a real data set to illustrate the flexibility of the new distribution. We estimated the model parameters using the bbmle package in R. The estimated values of the model parameters (standard error in parenthesis), $-2 \log$-likelihood statistics $(-2 \ln (L))$, Akaike Information Criterion (AIC), Bayesian Information Criterion (BIC), and Consistent Akaike Information Criterion (AICC) The Cramer-von Mises and Anderson-Darling goodness-of-fit statistics $W^{*}$ and $A^{*}$ described by (Chen and Balakrishnan, 1995) are also presented. These statistics can be used to verify which distribution fits better to the data. In general, the smaller the values of $W^{*}$ and $A^{*}$, the better the fit. The AdequacyModel package in R (R Development Core Team, 2011) was used to evaluate the fitted distributions.

We can use the likelihood ratio (LR) test to compare the fit of the KOL-LLo distribution with its sub-models for a given data set. For example, to test $a=b=1$, the LR statistic is $X^{2}=2[\ln (L(\hat{a}, \hat{b}, \hat{\lambda}, \hat{c}))-\ln (L(1,1, \tilde{\lambda}, \tilde{c}))]$, where $\hat{a}, \hat{b}, \hat{\lambda}$ and $\hat{c}$, are the unrestricted estimates, and $\tilde{\lambda}$, and $\tilde{c}$ are the restricted estimates. The LR test rejects the null hypothesis if $X^{2}>\chi_{d}^{2}$, where $\chi_{d}^{2}$ denote the upper $100 d \%$ point of the $\chi^{2}$ distribution with 2 degrees of freedom.

Plots of fitted densities and the histogram of the data are given. Probability plots (Chambers, Cleveland, Kleiner and Tukey, 1983) are also presented. Furthermore, values of the sum of squares $S S=\sum_{j=1}^{n}\left(F_{K O L-L L o}\left(x_{(j)}\right)-\frac{j-0.375}{n+0.25}\right)^{2}$ is presented. This statistic is used to compare the distributions and are given in the probability plots.

\subsection{Plasma Concentration Data}

The KOL-LLo distribution was fitted to the plasma concentration data. The data set was taken from R base package in the Indometh object. The fitted KOL-LLo model for plasma concentration data was compared to the fits of its nested models and four non-nested models, namely exponentiated Dagum (ED), gamma log-logistic (GLLo) and gamma Burr III (GB) distributions (See (Oluyede, Huang and Pararai, 2014), for details) and the exponentiated power generalized Weibull distributions (EPGW) ( Fernando, Peña-Ramírez, Renata, Cordeiro and Marinho, 2018) distributions. Gamma 
Table 3. Monte Carlo Simulation Results for KOL-LLo Distribution: Mean, RMSE, and Average Bias

\begin{tabular}{ccccc|ccc}
\hline & \multicolumn{3}{c|}{$\mathrm{a}=1.0, \mathrm{~b}=1.0, \lambda=0.5, \mathrm{c}=0.5$} & \multicolumn{3}{c}{$\mathrm{a}=1.5, \mathrm{~b}=1.0, \lambda=1.5, \mathrm{c}=0.5$} \\
\hline Parameter & $\mathrm{n}$ & Mean & RMSE & ABias & Mean & RMSE & ABias \\
\hline \multirow{4}{*}{$\mathrm{a}$} & 2.6349 & 8.3017 & 1.6349 & 3.9819 & 12.9437 & 2.4819 \\
& 50 & 1.4803 & 3.9877 & 0.4803 & 2.9864 & 9.3926 & 1.4864 \\
& 100 & 1.2737 & 1.4245 & 0.2737 & 2.0717 & 2.9127 & 0.5717 \\
& 200 & 1.1236 & 0.4537 & 0.1236 & 2.1418 & 3.6989 & 0.6418 \\
& 400 & 1.0835 & 0.6672 & 0.0835 & 1.7152 & 0.6810 & 0.2152 \\
& 800 & 1.0502 & 0.2657 & 0.0502 & 1.6487 & 0.4429 & 0.1487 \\
\hline \multirow{4}{*}{$\mathrm{b}$} & 1.8281 & 3.9829 & 0.8281 & 2.5395 & 5.0113 & 1.5395 \\
& 50 & 1.8001 & 7.4093 & 0.8001 & 2.3070 & 3.8336 & 1.3070 \\
& 100 & 1.5092 & 2.7894 & 0.5092 & 1.9999 & 2.5985 & 0.9999 \\
& 200 & 1.1897 & 1.0916 & 0.1897 & 2.0911 & 3.0978 & 1.0911 \\
& 400 & 1.1488 & 1.4498 & 0.1488 & 1.8573 & 2.0700 & 0.8573 \\
& 800 & 1.0671 & 0.8202 & 0.0671 & 1.4896 & 1.4803 & 0.4896 \\
\hline \multirow{4}{*}{25} & 0.8043 & 0.9427 & 0.3043 & 2.3694 & 2.7043 & 0.8694 \\
& 50 & 0.6473 & 0.6442 & 0.1473 & 2.2711 & 2.2672 & 0.7711 \\
& 100 & 0.6973 & 0.5497 & 0.1973 & 2.3289 & 1.9650 & 0.8289 \\
& 200 & 0.6494 & 0.4192 & 0.1494 & 2.1453 & 1.5588 & 0.6453 \\
& 400 & 0.6409 & 0.3910 & 0.1409 & 2.0393 & 1.4097 & 0.5393 \\
& 800 & 0.5780 & 0.2665 & 0.0780 & 2.0364 & 1.2270 & 0.5364 \\
\hline & 25 & 0.5630 & 0.2434 & 0.0630 & 0.7217 & 0.4646 & 0.2217 \\
& 50 & 0.5256 & 0.1537 & 0.0256 & 0.5880 & 0.2778 & 0.0880 \\
& 100 & 0.4964 & 0.1124 & -0.0036 & 0.5014 & 0.1606 & 0.0014 \\
& 200 & 0.4911 & 0.0859 & -0.0089 & 0.4714 & 0.1353 & -0.0286 \\
& 400 & 0.4964 & 0.0663 & -0.0036 & 0.4741 & 0.1050 & -0.0259 \\
& 800 & 0.4933 & 0.0481 & -0.0067 & 0.4839 & 0.0921 & -0.0161 \\
\hline
\end{tabular}

Dagum pdf (ution (Oluyede et al., 2014) is given by

$$
\begin{aligned}
f_{G D}(x) & =\frac{\lambda \beta \delta x^{-\delta-1}}{\Gamma(\alpha) \theta^{\alpha}}\left(1+\lambda x^{-\delta}\right)^{-\beta-1}\left(-\log \left(1-\left(1+\lambda x^{-\delta}\right)^{-\beta}\right)\right)^{\alpha-1} \\
& \times\left(1-\left(1+\lambda x^{-\delta}\right)^{-\beta}\right)^{(1 / \theta-1)} .
\end{aligned}
$$

When $\alpha=1, \beta=1$ and $\lambda=1$, GD distribution reduces to ED, GLLo ad GB distributions, respectively.

The pdf of EPGW is given by

$$
f(x ; \lambda, \gamma, \alpha, \beta)=\frac{\alpha \beta \lambda \gamma x^{\gamma-1}\left(1+\lambda x^{\gamma}\right)^{\alpha-1} \exp \left(1-\left(1+\lambda x^{\gamma}\right)^{\alpha}\right)}{\left(1-\exp \left(1-\left(1+\lambda x^{\gamma}\right)^{\alpha}\right)\right)^{(1-\beta)}},
$$

for $\lambda, \gamma, \alpha, \beta>0$, and $x>0$.

Table 4. Plasma Concentration Data

\begin{tabular}{llllllll}
\hline 1.50 & 0.94 & 0.78 & 0.48 & 0.37 & 0.19 & 0.12 & 0.11 \\
0.08 & 0.07 & 0.05 & 2.03 & 1.63 & 0.71 & 0.70 & 0.64 \\
0.36 & 0.32 & 0.20 & 0.25 & 0.12 & 0.08 & 2.72 & 1.49 \\
1.16 & 0.80 & 0.80 & 0.39 & 0.22 & 0.12 & 0.11 & 0.08 \\
0.08 & 1.85 & 1.39 & 1.02 & 0.89 & 0.59 & 0.40 & 0.16 \\
0.11 & 0.10 & 0.07 & 0.07 & 2.05 & 1.04 & 0.81 & 0.39 \\
0.30 & 0.23 & 0.13 & 0.11 & 0.08 & 0.10 & 0.06 & 2.31 \\
1.44 & 1.03 & 0.84 & 0.64 & 0.42 & 0.24 & 0.17 & 0.13 \\
0.10 & 0.09 & & & & & & \\
\hline
\end{tabular}


Table 5. Estimation of KOL-LLo Model for Plasma concentration Data

\begin{tabular}{|c|c|c|c|c|c|c|c|c|c|c|c|c|c|}
\hline \multirow[b]{2}{*}{ Distribution } & \multicolumn{4}{|c|}{ Estimates } & \multicolumn{5}{|c|}{ Statistics } & \multirow[b]{2}{*}{$A^{*}$} & \multirow[b]{2}{*}{$W^{*}$} & \multirow[b]{2}{*}{ K-S } & \multirow[b]{2}{*}{ p-value } \\
\hline & $\mathrm{a}$ & $\mathrm{b}$ & $\lambda$ & $c$ & - & $-2 \log \mathrm{L}$ & AIC & AICC & BIC & & & & \\
\hline KOL-LLo & $\begin{array}{l}517.33 \\
(0.0028)\end{array}$ & $\begin{array}{l}0.1219 \\
(0.0304)\end{array}$ & $\begin{array}{l}21.021 \\
(3.1561)\end{array}$ & $\begin{array}{l}0.4335 \\
(0.0536)\end{array}$ & - & 47.4501 & 55.4504 & 56.1062 & 64.2090 & 1.0071 & 0.1670 & 0.1169 & 0.3278 \\
\hline OL-LLo & $\begin{array}{l}1 \\
-\end{array}$ & $\begin{array}{l}1 \\
-\end{array}$ & $\begin{array}{l}2.2031 \\
(0.2201)\end{array}$ & $\begin{array}{l}0.9082 \\
(0.0874)\end{array}$ & - & 63.2221 & 67.2221 & 67.4126 & 71.6014 & 1.6437 & 0.2565 & 0.1328 & 0.1951 \\
\hline EOL-LLo & $\begin{array}{l}517.38 \\
(0.000487)\end{array}$ & $\begin{array}{l}1 \\
-\end{array}$ & $\begin{array}{l}8.6384 \\
(0.2513)\end{array}$ & $\begin{array}{l}0.1521 \\
(0.0138)\end{array}$ & - & 55.7314 & 61.7314 & 62.1185 & 68.3004 & 1.3993 & 0.2247 & 0.1305 & 0.2109 \\
\hline $\operatorname{KOL-LLo}(1,1, \lambda, 1)$ & $\begin{array}{l}1 \\
-\end{array}$ & $\begin{array}{l}1 \\
-\end{array}$ & $\begin{array}{l}2.2152 \\
(0.2208)\end{array}$ & $\begin{array}{l}1 \\
-\end{array}$ & - & 64.2739 & 66.2739 & 66.3364 & 68.4636 & 1.6882 & 0.2635 & 0.1654 & 0.0540 \\
\hline $\operatorname{KOL-LLo}(\mathrm{a}, 1, \lambda, 1)$ & $\begin{array}{l}0.9139 \\
(0.1509) \\
\end{array}$ & $\begin{array}{l}1 \\
- \\
\end{array}$ & $\begin{array}{l}2.1069 \\
(0.2915) \\
\end{array}$ & $\begin{array}{l}1 \\
- \\
\end{array}$ & - & 63.9725 & 67.9725 & 68.1630 & 72.3518 & 1.6919 & 0.2639 & 0.1478 & 0.1120 \\
\hline & $\alpha$ & $\beta$ & $\lambda$ & $\delta$ & $\theta$ & & & & & & & & \\
\hline ED & $\begin{array}{l}1 \\
-\end{array}$ & $\begin{array}{l}682.25 \\
(0.0000016)\end{array}$ & $\begin{array}{l}0.00127 \\
(0.00194)\end{array}$ & $\begin{array}{l}0.5510 \\
(0.4198)\end{array}$ & $\begin{array}{l}0.2984 \\
(0.5239)\end{array}$ & 56.3323 & 64.3323 & 64.9880 & 73.0909 & 1.4154 & 0.2261 & 0.1349 & 0.1811 \\
\hline GLLo & $\begin{array}{l}25.0582 \\
(0.00329)\end{array}$ & $\begin{array}{l}1 \\
-\end{array}$ & $\begin{array}{l}0.00435 \\
(0.00934)\end{array}$ & $\begin{array}{l}0.8095 \\
(0.3073)\end{array}$ & $\begin{array}{l}0.1814 \\
(0.0714)\end{array}$ & 55.5925 & 63.5925 & 64.2483 & 72.3512 & 3.0998 & 0.5278 & 1 & $<2.2 \times 10^{-16}$ \\
\hline GB & $\begin{array}{l}38.095 \\
(0.00002) \\
\alpha\end{array}$ & $\begin{array}{l}1.6327 \\
(0.000686) \\
\beta\end{array}$ & $\begin{array}{l}1 \\
- \\
\lambda\end{array}$ & $\begin{array}{l}0.1377 \\
(0.0120) \\
\delta\end{array}$ & $\begin{array}{l}0.00882 \\
(0.000207) \\
\gamma\end{array}$ & 56.5533 & 64.5533 & 65.2090 & 73.3119 & 2.8612 & 0.4783 & 1 & $<2.2 \times 10^{-16}$ \\
\hline EPGW & $\begin{array}{l}0.3994 \\
(0.00569)\end{array}$ & $\begin{array}{l}253.35 \\
(0.00000861)\end{array}$ & $\begin{array}{l}198.05 \\
(0.00000898)\end{array}$ & $\begin{array}{l}1 \\
-\end{array}$ & $\begin{array}{l}0.3747 \\
(0.03013)\end{array}$ & 55.7284 & 63.7284 & 64.3842 & 72.4871 & 1.4014 & 0.2252 & 0.1301 & 0.2137 \\
\hline
\end{tabular}

The variance covariance matrix is given by

$$
\left[\begin{array}{cccc}
0.00000797 & -0.0000743 & 0.008915 & 0.000141 \\
-0.0000743 & 0.000921 & -0.08306 & -0.00128 \\
0.008915 & -0.08306 & 9.9610 & 0.1579 \\
0.000141 & -0.00128 & 0.1579 & 0.00288
\end{array}\right]
$$

and the $95 \%$ interval estimates for the parameters are $a \in[517.3245,517.3355], b \in[0.0624,0.1814], \lambda \in[14.8530,27.2069]$ and $c \in[0.3283,05387]$.

Likelihood ratio test results are shown in Table 6. From results of the likelihood ratio test, we conclude that there are

Table 6. Likelihood ratio test results for plasma concentration data

\begin{tabular}{cccc}
\hline Model & Chi-Square & df & p-value \\
\hline OL-LLo & 15.8 & 2 & 0.00038 \\
EOL-LLo & 8.3 & 1 & 0.00410 \\
KOL-LLo $(1,1, \lambda, 1)$ & 16.8 & 3 & 0.00077 \\
KOL-LLo $(1, \mathrm{~b}, \lambda, 1)$ & 15.7 & 2 & 0.00038 \\
KOL-LLo(a,1, $\lambda, 1)$ & 16.5 & 2 & 0.00043 \\
\hline
\end{tabular}

significant differences between the KOL-LLo distribution and the nested models. Also based on the lowest values of the goodness-of-fit statistics $A^{*}, W^{*}$ and K-S statistic and bigger p-value, the KOL-LLo model fits the data better than the ED, GLLo, GB and EPGW models.

\section{Concluding Remarks}

We have developed a new family of distributions, namely Kumaraswamy odd Lindley-G, which is a generalization of the odd Lindley-G distribution. We derived the statistical properties of the new family of distributions. The KOL-G new family can be expressed as a mixture of Ex-G densities. Special cases were also presented, namely, KOL-U, KOL-LLo and KOL-W distributions. Application of the KOL-LLo distribution to a real data set shows that it performed better than the nested models and non-nested ED, GLLo, GB and EPGW distributions for the plasma concentration data.

\section{Acknowledgments}

We are grateful to the referees and the editor for their comments which greatly improved this paper. 


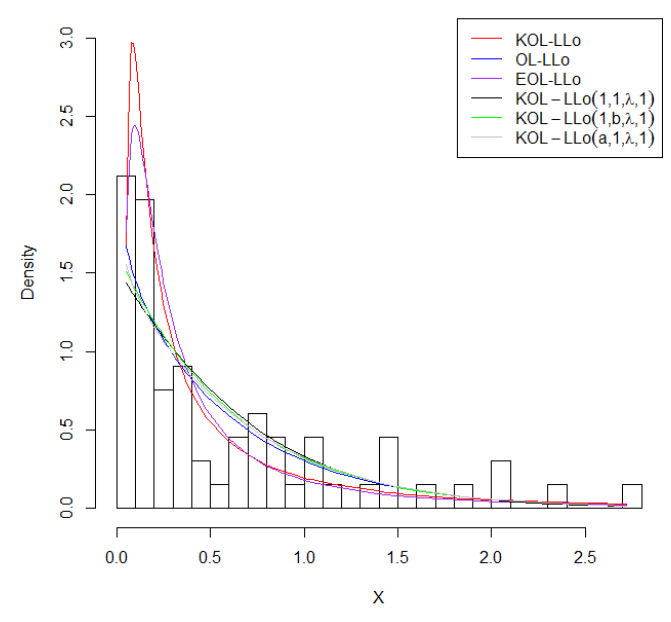

Figure 7. Fitted PDFs for plasma concentration data

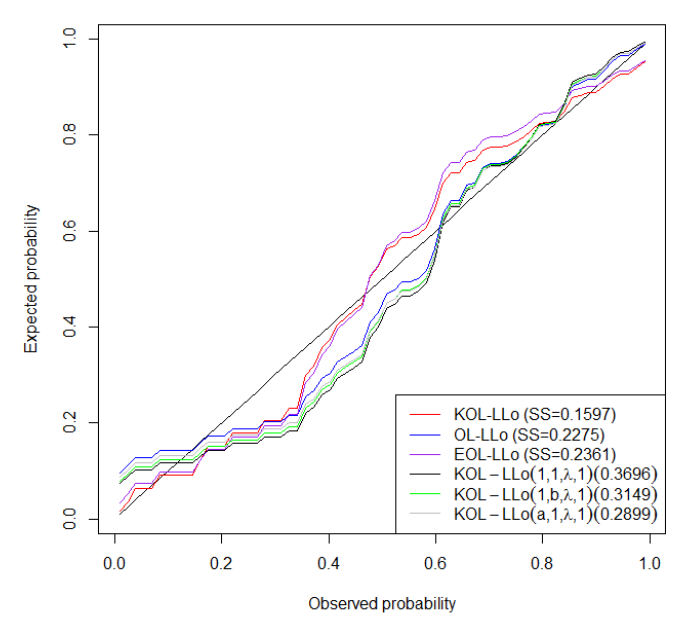

Figure 8. Observed Probabilities for KOL-LLo model for plasma concentration data

\section{References}

Alexander, C., Cordeiro, G. M., Ortega, E. M. M., \& Sarabia, J. M. (2012). Generalized beta-generated distributions. Computational Statistics and Data Analysis, 56, 1880-1897. https://doi.org/10.1016/j.csda.2011.11.015

Alzaatreh, A., Lee, C., \& Famoye, F. (2013). A new method for generating families of continuous distributions. Metron, 71, 63-79. https://doi.org/10.1007/s40300-013-0007-y

Alzaghal A., Famoye, F., \& Lee, C. (2013). Exponentiated T-X Family of distributions with some applications. International Journal of Statistics and Probability, 2, 31-49. https://doi.org/10.5539/ijsp.v2n3p31

Bourguignon, M., Silva, R. B., \& Cordeiro, G. M. (2014). The Weibull-G family of probability distributions. Journal of Data Science, 12, 53-68.

Chambers, J., Cleveland, W., Kleiner, B., \& Tukey, J. (1983). Graphical Methods for Data Analysis. London, Chapman and Hall.

Chen, G., \& Balakrishnan, N. (1995). A General Purpose Approximate Goodness-of-fit Test, Journal of Quality Technology, 27, 154-161. https://doi.org/10.1080/00224065.1995.11979578 
Cordeiro, G. M., Ortega, E. M. M., Popović, B. V., \& Pescim, R. R. (2014). The Lomax generator of distributions: properties, minification process and regression model. Applied Mathematics and Computation, 247, 465-486.

Cordeiro, G. M., Gomes, A., da Silva, C., \& Ortega, E. M. M. (2013). The beta exponentiated Weibull distribution. Journal of Statistical Computation and Simulations, 38(1), 114-138. https://doi.org/10.1080/00949655.2011.615838

Cordeiro, G. M., \& de Castro, M. (2011). A new family of generalized distributions. Journal of Statistical Computation and Simulation, 81(7), 883-898. https://doi.org/10.1080/00949650903530745

Eugene, N., Lee, C., \& Famoye, F. (2002). Beta-normal distribution and its applications. Communications in StatisticsTheory and Methods, 31, 497-512. https://doi.org/10.1081/STA-120003130

Fernando, A., Peña-Ramírez, Renata, R. G., Cordeiro, G. M., \& Marinho, P. R. D. (2018). The exponentiated power generalized Weibull: Properties and Applications. Annals of the Brazilian Academy of Sciences, 90(23), 2553-2577.

Gomes-Silva, F., Percontini, A., de Brito, E., Ramos, M. W., Venáncio, R., \& Cordeiro, G. M. (2017). The odd Lindley-G family of distributions. Austrian Journal of Statistics, 46, 65-87.

Jones, M. C. (2009). Kumaraswamy's distribution: a beta-type distribution with tractability advantages. Statistical Methodology, 6, 70-81. https://doi.org/10.1016/j.stamet.2008.04.001

Kumaraswamy, P. (1980). Generalized probability density function for double-bounded random process. Journal of Hydrology, 46, 79-88. https://doi.org/10.1016/0022-1694(80)90036-0

Makubate, B., Oluyede, B. O., Motobetso, G., Huang, S., \& Fagbamigbe, A. F. (2018). The beta Weibull-G family of distributions: model, properties and application. International Journal of Statistics and Probability, 7(2), 12-32.

Nadarajah, S., \& Kotz, S. (2006). The exponentiated-type distributions. Acta Applicandae Mathamaticae, 92, 97-111. https://doi.org/10.1007/s10440-006-9055-0

Nadarajah, S., \& Kotz, S. (2004). The beta Gumbel distribution. Mathematical Problems in Engineering, 4, 323-332. https://doi.org/10.1155/S1024123X04403068

Oluyede, B. O., \& Yang, T. (2015). A new class of generalized Lindley distributions with applications. Journal of Statistical Computation and Simulation, 85(10), 2072-2100.

Oluyede, B. O., Huang, S., \& Pararai, M. (2014). A new class of generalized Dagum distributions with applications to income and lifetime data. Journal of Statistical and Econometric Methods, 3(2),125-151.

Peña-Ramírez, F. A., Guerra, R. R., Cordeiro, G. M., \& Marinho P. R. D. (2018). The exponentiated power generalized Weibull: Prpoerties and Applications. Annals of the Brazilian Academy of Sciences, 90(23), 2553-2577.

R Development Core Team, A Language and Environment for Statistical Computing, R Foundation for Statistical Computing, Vienna, Austria, (2011).

Rényi, A. (1961). On the measure of entropy and information. Berkeley Symposium on Mathematical Statistics and Probability, 1, 547-561.

Ristić, M. M., \& Balakrisihnan, N. (2012). The gamma exponentiated exponential distribution. Journal of Computation and Simulation, 82, 1191-1206.

Shannon, C. E. (1951). Predection and entropy of printed english. The Bell System Technical Journal, 30, 50-64. https://doi.org/10.1002/j.1538-7305.1951.tb01366.x

Torabi, H., \& Montazari, H. N. (2012). The gamma-uniform distribution and its applications. Kybernetika, 48(1), 16-30.

Zografos, K., \& Balakrishnan, N. (2009). On families of beta- and generalized gamma-generated distributions and associated inference. Statistical Methodology, 6, 344-362. https://doi.org/10.1016/j.stamet.2008.12.003

\section{Copyrights}

Copyright for this article is retained by the author(s), with first publication rights granted to the journal.

This is an open-access article distributed under the terms and conditions of the Creative Commons Attribution license (http://creativecommons.org/licenses/by/4.0/). 